\title{
Numerical Simulation of Spiral Flow and Heat Transfer in Hydrate Pipeline With Long Twisted Band
}

\section{Yongchao Rao}

Changzhou University

Lijun Li

Changzhou University

Shuli Wang ( $\nabla$ wsl@cczu.edu.cn )

Changzhou University

Shuhua Zhao

Changzhou University

Shidong Zhou

Changzhou University

\section{Research Article}

Keywords: DPM model (discrete phase model), pressure drop distribution, straight light pipe flow, Spiral flow

Posted Date: December 9th, 2020

DOl: https://doi.org/10.21203/rs.3.rs-118456/v1

License: (9) (1) This work is licensed under a Creative Commons Attribution 4.0 International License. Read Full License 


\title{
Numerical Simulation of Spiral Flow and Heat Transfer in Hydrate Pipeline with Long Twisted Band
}

\author{
Yongchao Rao ${ }^{1,2}$, Lijun $\mathrm{Li}^{1,2}$, Shuli Wang ${ }^{1,2^{*}}$, Shuhua Zhao ${ }^{1,2}$, Shidong Zhou ${ }^{1,2}$
}

The DPM model (discrete phase model) considering the motion of solid particles was used to simulate the complex spiral flow characteristics of hydrate in the pipe spinning up with long twisted band. The deposition and heat transfer characteristics of gas hydrate particles in the pipe spiral flow were studied. The velocity distribution, pressure drop distribution, heat transfer characteristics and particle settling characteristics of the flow field in the pipeline were investigated. The numerical results show that, compared with the straight flow of light pipe without twisted band, two obvious eddies are formed in the flow field under the spinning action of twisted band, and the velocities are maximum at the center of the eddies. Along the direction of the pipe, the two vortices move towards the pipe wall from near the twisted band, which can effectively carry the hydrate particles deposited on the pipe wall. With the same Reynolds number, the greater the twist ratio, the weaker the spiral strength, the smaller the tangential velocity of the spiral flow, and the smaller the pressure drop of the pipe. Therefore, the pressure loss can be reduced as much as possible while ensuring the spinning effect of the spiral flow. In a straight light pipe flow, the Nusser number is in a parabolic shape with the opening downwards. At the center of the pipe, the Nusser number gradually decreases towards the pipe wall at the maximum, and at the near wall, the attenuation gradient of $\mathrm{Nu}$ is large. For spiral flow, the curve presented by Nusserr number shows a trough at the center of the pipe and a peak at 1/2 of the pipe diameter. With the reduction of twist rate, the Nussel number becomes larger and larger. Therefore, the spiral flow can make the temperature distribution in the flow field in the pipeline more even, and prevent the large temperature difference resulting in the mass formation of hydrate particles in the pipeline wall. Spiral flow has a good carrying effect. Under the same working condition, the spiral flow carries hy drate particles at a distance about 3-4 times that of the straight flow.

${ }^{1}$ School of Petroleum Engineering, Changzhou University, Changzhou 213164, China. ${ }^{2}$ Key Laboratory of Oil and Gas Storage and Transportation Technology of Jiangsu Province, Changzhou 213164, China

* e-mail: wsl@cczu.edu.cn

\section{Introduction}

Mixed pipeline of natural gas hydrate plugging problems more and more serious, hydrate blocka ge blocks the flow of natural gas makes the partial pressure of pipeline damage to pipeline equipment, control hydrate plugging gradually become an important problem to ensure the safety of pipeline operation. The traditional methods to prevent hydrates from blocking pipelines are to change the formation conditions of hydrates by heating and lowering the pressure, or to inhibit the formation of hydrates by injecting thermodynamic inhibitors. These methods not only cost a lot but also cause pollution to the environment. The current research is not foc used on hydrate suppression but on the generation of hydrate to ensure the safe flow of hydrate to achieve the purpose of risk control.

There are many kinds of spiral rotating devices, such as spiral twisted band, impeller and spiral guide bar. According to the different use conditions and purposes need to use different rotating device, there are three methods for the conventional generation of spiral flow: tangential in let flow, installation of flow guide and spiral pipe. Wang et al. ${ }^{1-2}$ made a comparative analysis of various spiral flow rotating devices and carried out experimental studies. Team $^{3}$ used PHOENICS computational fluid softwa re to numerically simulate the formation and attenuation of spiral flow in a pipeline with a velocity angle of $5 \sim 70^{\circ}$. It is found that when the velocity angle changes between $5^{\circ}$ and $30^{\circ}$, the resulting spiral flow has a suitable tangential and axial velocity, which is also conducive to the removal of deposited impurities and water in the pipeline. Dou et al. ${ }^{4}$ analyzed the liquid-solid two-phase flow in the tube with built-in spring. Compared with the flow in the non-helical tube, the helical flow not only enhanced heat transfer, but also had the effect of cleaning dirt. Zhang et al. ${ }^{5}$ studied the attenuation characteristics of the spiral flow of the flat axis round tube generated by the local generator through experiments, and he concluded that the tangentialvelocity and the strength of the spiral flow decreased with the increase of the distance. In conclusion, spiral flow not only has strong carry ing capacity, but also has important application value for safe transportation of NGH. The research group also used the RNG k- $\varepsilon$ model to numerically simulate the flow characteristics of swirl flow rotated by the vane in the 
horizontal pipe ${ }^{6}$. The Reynolds number had a great influence on swirl intensity. The swirl intensity would increase, and swirl intensity attenuation rate decrease, when Re number increased. Moreover, the spiral flow can enhance the heat transfer between phases, which is a controllable technique for the formation and prevention of hydrate in the pipeline.

Research on the safe flow of natural gas hydrate, Zhao et al. ${ }^{7}$ considered the interaction among hydrate, particle size distribution, particle viscosity, the biggest internal phase accumulation rate of the basic physical properties, such as based on E-Euler double fluid model, the mathematical model of hydrate flow in pipeline was established, the results of the study showed that compared with Mulhe size model, the particle size of model considered the interaction between the hydrate particles, the simulation value is closer to the experimental value. Song et al. ${ }^{8}$ introduced a population equilibrium model based on the agregation dynamics of hydrate particles to simulate the effects of fluid flow rate and hydrate volume fraction on the safe flow characteristics of hydrate slurry. Then the population balance model based on solid hydrate grain accumulation dynamic ${ }^{9}$, the collision frequency, aggregation efficiency, fragmentation frequency and sub-particles size distribution function after crushing of the hydrate solid particles were considered in the flow process, simulation under different conditions of hydrate particle aggregation process, the simulation results were compared with the calculated results of the hydrate particle growth model. In addition, Song et al. ${ }^{10-11}$ simulated the deposition rule of gas hydrate particles in the pipeline by referencing condensation method, and established the kinetic model of hydrate deposition and hydrate decomposition. Based on the theory of hydrate particle deposition, the structural model of hydrate deposition was established, the main mechanical parameters were determined, and the finite element numerical simulation method was used to calculate the hydrate deposition on the pipe wall. Song et a $1 .{ }^{12}$ established a dynamic model of hydrate aggregation based on the population equilibrium equation. From the dy namic model, they gave and fully discussed the calculation method of particle fragmentation and collision frequency, generation condensation efficiency, fra gmentation frequency and particle size distribution function of sub-particles. The traditional solid-liquid flow model is solved, the effects of flow rate and hydrate volume fraction on hydrate slurry flow characteristics in pipelines of oil and water systems were simulated. Liang Jun et al. ${ }^{13-14}$ th rough the establishment of three-dimensional model of circular pipe spiral flow, RNG k- $\varepsilon$ turbulence model and DPM model were used to simulate the three-dimensionaltransient of gas-solid twophase spiral flow and heat transfer in natural gas pipeline, the velocity field, temperature field, distribution law of hydrate particle volume fraction and heat transfer law of different cross sections in natural gas pipeline were studied. And experimental research on characteristics of the gas-solid two phase flow spun up by the twisted band in pipelines is conducted ${ }^{15}$, and the effect of gas phase velocity and parameters of twisted band on the particle carrying laws, collision characteristics, carrying distance, particle velocity distribution and particle concentration distribution is investigated, and flow characteristics of gas-solid two phase non-spiral flow and gas-solid two phase spiral flow are compared and analyzed. Cai et al. ${ }^{16}$ used the DPM model and RSM model to simulate the spiral transport of gas hydrate particles with a twisted band. The temperature field, velocity field, turbulence intensity and deposition law of hydrate particles under different torsion rates and flow rates were examined. Chang et al. ${ }^{17}$ carried out three dimensional transient numerical simulation and simulation of gas-solid two-phase spiral flow in natural gas pipelines, and studied the volume concentration variation characteristics of hydrate particles in natural gas pipelines and the deposition characteristics of hydrate particles. Considering the formation process of hydrate, Liu et al. ${ }^{18}$ established the equation of mass, momentum and energy balance. The iterative method and finite difference method are used to solve the model results, and the sensitivity analysis of the important parameters of the model is carried out. Brown et al. ${ }^{19}$ used a micromechanical force meter to explore the interaction between the wax deposited on the surface and dissolved in the bulk phase and the hydrate chemically treated to prevent agglomeration. It is found that wax can sign if icantly change the cohesion and adhesion of hydrate particles, but the effect of anti-caking agent may vary with the composition of anti-caking agent. Nicholas et a.$^{20}$ explored the deposition of saturated natural gas condensate containing water on the pipeline wall by using single-circulation pipeline, and found that the deposition of hydrate on the wall could cause the pipeline pressure drop to rise slowly. At the same time, the wall sediments can insulate the fluid inside the tube, making the wall sediments migrate downstream. Lorenzo et al. ${ }^{21}$ studied the growth and deposition of hydrate on the wall under the condition of annular flow with single circulation pipeline, and found that the membrane growth model under the condition of low subcooling could well predict the pressure drop of the loop after hydrate formation. However, other particle behavior characteristics, such as the shedding of hydrate layer, should be considered under the condition of high supercooling.

In conclusion, academics have carried out the numerical simulation and experimental research of the combination of pipeline flow law of gas hydrate in security work, but it did not hydrate particles in a long twist band unscrew the system research to the movement of the sedimentary rules, at the same time and heat transfer of the spiral flow system of the law on hydrate formation and the influence of the particle movement is also the important interf erence factors of the pipeline operation. Therefore, in this paper, the author in view of the current hydrate flow characteristics of common flow field, the DPM model (discrete phase model) that considers the movement of solid 
particles is used to numerically simulate the complex spiral flow characteristics of hydrates inside a pipe swirling with a long twisted band, focus on the deposition and heat transfer characteristics of natural gas hydrate particles in pipe spiral flow. Investigate the velocity distribution, pressure drop distribution, heat transfer characteristics and particle settling characteristics of the flow field in the pipeline. Provide technical support for the safe transportation of natural gas hydrate in pipelines under spiral flow system.

\section{Numerical simulation method}

\section{1 physical model}

The formation of hydrate requires a series of complex processes and does not ex ist directly in the pipeline. In this paper, a series of reaction processes of hydrate formation are ignored in the numerical simulation, and it is assumed that hydrate particles have been generated directly at the entrance of the pipeline. In the numerical simulation, the shape of hydrate particles was set as a positive circle and the particle size was also set as the same, while the influence of pipe wall thickness on the formation of hydrate was directly ignored in the calculation. The calculation model established by CAD softwa re is shown in Fig. 1 . The pipe diameter $\mathrm{D}=0.024 \mathrm{~m}$ and the length $\mathrm{L}=2.5 \mathrm{~m}$. At the same time, a group of hydrate particles with partial rotation in the short twisted band was set as a comparison, and the length of the short twisted band was $0.5 \mathrm{~m}$.

\subsection{1 geometric model}

Twisted band with different torsional rates a re set at the pipe inlet as the swirler. The physical model of the twisted band is shown in Fig.2, the torsional rates of the spiral twisted band are 6.2, 7.4 and 8.8 , respectively. Fig. 3 shows a schematic diagram of the torsional rate of the twisted band. The torsional rate is the ratio between the length $\mathrm{H}$ of the twisted band and the width $\mathrm{D}$ of the twisted band after a rotation.

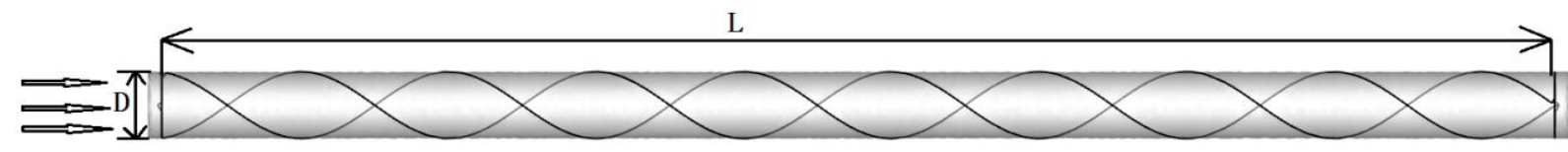

Figure 1. The physical model

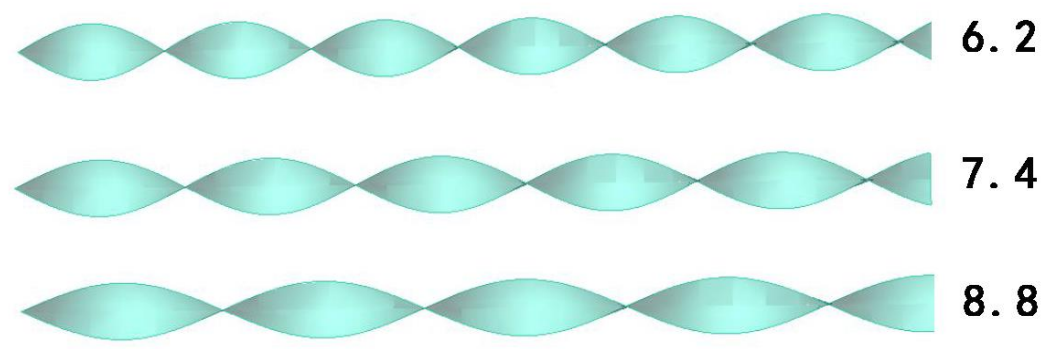

Figure 2. Twisted band model

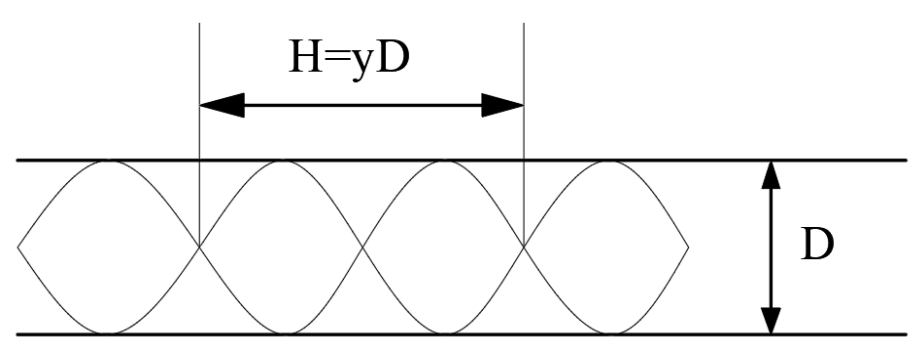

Figure 3. Twisted rate schematic diagram of twisted band

\subsection{2 boundary conditions}

Before numerical simulation, the boundary conditions of the geometric model should be set reasonably. In the inlet end of the pipe, the velocity inlet is set as the boundary condition. The velocity inlet for four different reynolds numbers is 5000, 10000, 15000 and 20000 respectively. In the outlet is setting outflow exports as the boundary conditions, no slip wall is set to a fixed, the problem is simplified to three dimensional, constant physical gas -solid flow. Set the inlet temperature $\mathrm{T}$ in of the pipeline to $280 \mathrm{~K}$, and the wall temperature $\mathrm{T}_{\mathrm{w}}=277 \mathrm{~K}$. The $\mathrm{model}$ is 
calculated using rectangular coordinate system. The origin is at the center of the entry interface, the $\mathrm{z}$-ax is is the flow direction, the gravity direction is along the -y axis, and the gravity acceleration is $9.81 \mathrm{~m} / \mathrm{s} 2$. The fluid medium is natural gas and hydrate particles, flowing from the left end of the pipeline to the right.

\subsection{3 initial conditions}

The diameter of the gas pipeline $\mathrm{D}=0.024 \mathrm{~m}$, and the length $\mathrm{L}=2.5 \mathrm{~m}$.In the simulation, it is considered that the physical properties of the hydrate in the spiral flow pipeline are constant, the gas phase is methane, and the solid phase is NGH particles. The basic physical parameters are measured at room temperature. Firstly, the natural gas density was set as $0.77 \mathrm{~kg} / \mathrm{m} 3$, the kinematic viscosity of natural gas was $11.03 \times 10-6 \mathrm{~m} 2 / \mathrm{s}$, and the hydrate particle density was $915 \mathrm{~kg} / \mathrm{m} 3$. The initial concentration of hydrate was given as $1 \%, 2 \%, 4 \%, 6 \%$ and $8 \%$ in DPM, and the movement of particles at different concentrations was simulated. The temperature of the fluid and hydrate particles in the natural gas pipeline are the same, and there is no temperature difference. Moreover, the hydrate particles are set as homogeneous spheres with the same particle size in the simulation. Data parameters are selected according to the research content, as shown in Table 1.

Table 1. Table of experimental parameters for numerical simulation

\begin{tabular}{cccc}
\hline $\begin{array}{c}\text { particle } \\
\begin{array}{c}\text { concentration } \\
(\%)\end{array}\end{array}$ & $\begin{array}{c}\text { particle } \\
\text { diameter } \\
(\mathrm{mm})\end{array}$ & $\begin{array}{c}\text { Twist with } \\
\text { twist rate Y }\end{array}$ & $\begin{array}{c}\text { Reynolds } \\
\text { number Re }\end{array}$ \\
\hline 1 & 0.01 & 0 & 5000 \\
2 & 0.02 & 6.2 & 10000 \\
4 & 0.03 & 7.4 & 15000 \\
6 & 0.06 & 8.8 & 20000 \\
\hline
\end{tabular}

\section{2 meshing}

Fig. 4 shows the calculation grid of the pipe section of the long twisted band. The entire geometric model is meshed. Because of the existence of the twisted band, the unstructured grid is used, and the dense processing of the pipe wall can better perform the turbulence calculation. The most suitable grid computing was selected through the grid independence test, and the number of grid cells was about 4 million.

\section{3 mathematical model}

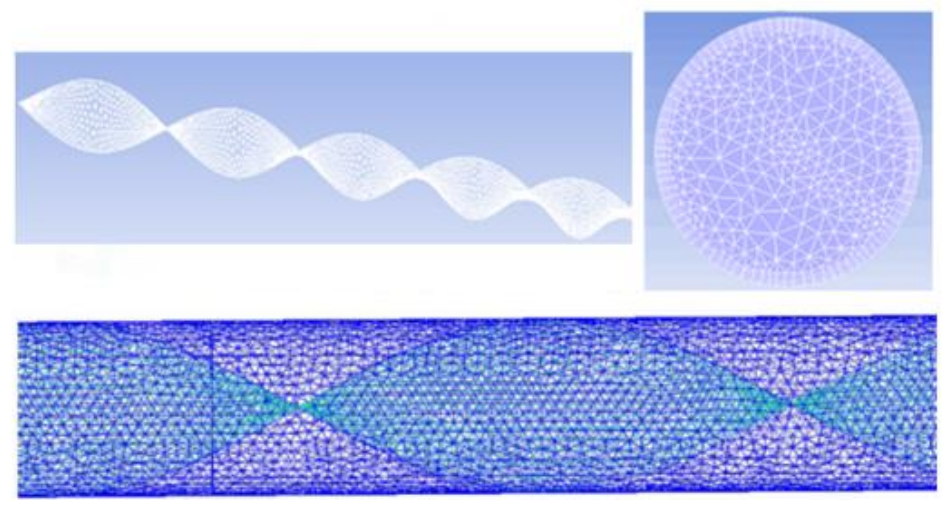

Figure 4. Calculation grid for long twisted band pipe section

The DPM model (discrete phase model) is used in the simulation. The discrete phase model is a multi-component flow model under the eula-la grangian method. In the simulation, the solid particles are treated as discrete phase, while the fluid is treated as continuous phase. After the fluid continuous phase is stabilized in the calculation, the particle discrete phase is introduced. In the discrete phase model, the particle phase is regarded as the discrete phase, while the fluid is only regarded as the continuous phase. The particle volume fraction is the mass transfer from phase $q$ to phase $\mathrm{p}$.

energy equation:

$$
\frac{\partial(\rho T)}{\partial \mathrm{t}}+\frac{\partial(\rho u T)}{\partial \mathrm{x}}+\frac{\partial(\rho v T)}{\partial \mathrm{y}}+\frac{\partial(\rho w T)}{\partial \mathrm{z}}=\frac{\partial}{\partial x}\left(\frac{\lambda}{\mathrm{c}_{p}} \frac{\partial T}{\partial x}\right)+\frac{\partial}{\partial \mathrm{y}}\left(\frac{\lambda}{\mathrm{c}_{p}} \frac{\partial T}{\partial y}\right)+\frac{\partial}{\partial z}\left(\frac{\lambda}{\mathrm{c}_{p}} \frac{\partial T}{\partial z}\right)
$$

Where, $\rho, c_{p}, T$ and $\lambda$ are gas density, specific heat capacity at constant pressure, temperature and thermal conductivity $\left(c_{p}=2.205 \mathrm{~kJ} / \mathrm{kg} \cdot \mathrm{k}, \lambda=0.14 \mathrm{~W} / \mathrm{m} \cdot \mathrm{k}\right), \mathrm{u}, \mathrm{v}$ and $\mathrm{w}$ are all velocities, and $\mathrm{t}$ is time. 


\subsection{1 governing equations}

equation of continuity

$$
\frac{\partial \rho}{\partial t}+\frac{\partial}{\partial x_{i}}\left(\rho u_{i}\right)=0
$$

momentum equation

$$
\frac{\partial}{\partial t}\left(\rho u_{i}\right)+\frac{\partial}{\partial x_{j}}\left(\rho u_{i} u_{j}\right)+\frac{\partial p}{\partial x_{i}}-\frac{\partial \tau_{i j}}{\partial x_{j}}-\frac{\partial \tau_{i j}^{-1}}{\partial x_{j}}=0
$$

Where, $\rho, \mathrm{u}$, and $\mathrm{p}$ are gas density, velocity, and static pressure, respectively; $\tau_{i j}$ is the viscous stress tensor; $\mathrm{t}$ is the time. Volume fraction equation:

$$
\frac{1}{\rho_{q}}\left[\frac{\partial}{\partial t}\left(a_{q} \rho_{q}\right)+\nabla \cdot\left(a_{q} \rho_{q} \bar{v}_{q}\right)=S_{a_{q}}+\sum_{p=1}^{n}\left(\dot{m}_{p q}-\dot{m}_{q p}\right)\right]
$$

Where, $\mathrm{a}_{\mathrm{q}}$ is the volume fraction of phase $\mathrm{q}, S_{a_{q}}$ is the source term, $\dot{m}_{p q}$ is the mass transfer from phase p to phase $\mathrm{q}$, $\dot{m}_{q p}$ is required to be relatively small, and the solid particle concentration of the discrete phase is generally below $10 \%$.

2.3.2 turbulent motion equation

The standard $\mathrm{k}-\varepsilon$ model is a semi-empirical formula. The equation is:

$$
\begin{gathered}
\frac{\partial}{\partial \mathrm{t}}(\rho k)+\frac{\partial}{\partial x_{i}}\left(\rho k u_{i}\right)=\frac{\partial}{\partial x_{j}}\left[\left(\mu+\frac{\mu_{t}}{\sigma_{k}}\right) \frac{\partial k}{\partial x_{j}}\right]+\mathrm{G}_{k}-Y_{k}+S_{k} \\
\frac{\partial}{\partial \mathrm{t}}(\rho \varepsilon)+\frac{\partial}{\partial x_{i}}\left(\rho \varepsilon u_{i}\right)=\frac{\partial}{\partial x_{j}}\left[\left(\mu+\frac{\mu_{t}}{\sigma_{k}}\right) \frac{\partial \varepsilon}{\partial x_{j}}\right]^{\mathrm{C}_{1 \varepsilon} \frac{\varepsilon}{k}\left(G_{k}+C_{3 \varepsilon G_{b}}\right)-C_{2 \varepsilon} \rho \frac{\varepsilon^{2}}{k}+S_{\varepsilon}}
\end{gathered}
$$

Where:

$$
\mu_{t}=\rho C_{\mu} \frac{k^{2}}{\varepsilon}
$$

Among: $C_{1 \varepsilon}=1.44, C_{2 \varepsilon}=1.92, C_{\mu}=0.09, \sigma_{k}=1.0, \sigma_{\varepsilon}=1.3$.

\subsection{3 discrete phase model}

The calculation of discrete phase model mainly consists of continuous phase and dispersed phase. The DPM model can obtain the particle motion equation by calculating the forces on particles, and the particle motion trajectory can be obtained by integrating the particle motion equation of DPM model with time. The motion equation of the trajectory of solid particles in the $\mathrm{z}$ direction is:

$$
\frac{d_{u_{p}}}{d_{t}}=F_{D}\left(u-u_{p}\right)+\frac{g_{\mathrm{z}}\left(\rho_{p}-\rho\right)}{\rho_{p}}+F_{z}
$$

Where, $\mathrm{u}$ is the velocity of the fluid phase, $\mathrm{m} / \mathrm{s} ; u_{p}$ is particle velocity, $\mathrm{m} / \mathrm{s} ; g_{z}$ is the component of the gravitational acceleration in the $\mathrm{z}$ direction, $\mathrm{m} / \mathrm{s}^{2} ; \rho$ is the fluid density, $(\mathrm{kg} / \mathrm{m} 3) ; \rho_{p}$ is the particle density $\left(\mathrm{kg} / \mathrm{m}^{3}\right)$.The particles are mainly subjected to other forces $F_{z}$ including additional mass forces, Brownian forces, and Staffman lift forces et al. caused by fluid acceleration around the particles. The additional mass forces are mainly applied when the density of the fluid phase is greater than that of the particles. The particle density of hydrate in the pipeline is greater than that of the airflow, so it is not necessary to consider the additional mass forces.

Where, $F_{D}$ is defined as:

$$
F_{D}=\frac{18 \mu}{\rho_{p} d_{p}^{2}} \frac{C_{D} \operatorname{Re}}{24}
$$

Re is defined as the relative Reynolds number as follows: 


$$
\operatorname{Re}=\frac{\rho d_{p}\left|u_{p}-u\right|}{\mu}
$$

Where, $d_{p}$ is particle diameter, $\mathrm{m}$.

The drag coefficient $C_{D}$ can be expressed as follows:

$$
C_{D}=a_{1}+\frac{a_{2}}{\operatorname{Re}}+\frac{a_{3}}{\operatorname{Re}}
$$

$a_{1}, a_{2}$ and $a_{3}$ to constant values are as follows: $5000<\operatorname{Re}<10000$ are respectively $0.46,-490.546$, 578700; When $\operatorname{Re}>10000$, it is $0.5191,-16625.5$ and 5416700 respectively.

\section{4 calculation method}

CFD software is usually used to solve practical problems in numerical simulation. The software is generally composed of three parts: pretreatment, calculation and data solving, and post-processing. Pre-processing is to build a physical model and then conduct mesh division. The computing part in the middle is particularly important. Many parameters and algorithm selection need to be set before re-operation, among which the selection of algorithm can improve the accuracy of simulation and computing efficiency. The calculation can be roughly divided into the following steps: physical model selection, parameter setting (grid unit selection, material definition, phase selection, boundary condition setting, reference value setting, etc.), algorithm selection and control factor setting, initialization, monitor setting, and time step setting.

The discrete phase model is selected in the calculation, and the pressure base and implicit solver are used to simulate the transient state of the gas-solid three-dimensional helical flow in the gas hydrate pipeline. In the simulated calculation for discrete phase, with RNG k- $\varepsilon$ turbulence model, the SIMPLEC algorithm is adopted to the coupling of pressure and velocity, The discrete phase model is selected in the calculation, and the pressure base and implicit solver are used to simulate the transient state of the gas-solid three-dimensional helical flow in the gas hydrate

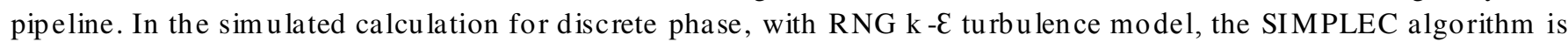
adopted to the coupling of pressure and velocity, a multidimensional linear reconstruction method is used to reconstruct the second order surface pressure scheme, particle motion model using DPM model. In the DPM model, it is also necessary to set some parameters at the in let of the pipeline, such as the injection particle speed, mass flow rate and injection particle number, etc. During the iterative calculation in the simulation, in order to calculate the effect of better, also need to set down the relaxation factor numerical range: $\varepsilon_{p}=0.3 \sim 0.7, \varepsilon_{m}=0.5 \sim 0.7, \varepsilon_{k}=\varepsilon_{\varepsilon}=0.4 \sim 0.6$. When the absolute value of residual is below $1 \times 10^{-6}$, the condition of convergence can be achieved.

\subsection{Numerical examples validate}

\subsection{1 grid independence test}

Mesh generation is an important step in the establishment of finite element model. In this process, many problems need to be considered and the workload is very heavy. The unit length, quantity and density of the grid have direct influence on the precision and time of calculation.

In order to meet the requirements of computational accuracy and ensure computational efficiency,8m m, $4 \mathrm{~mm}$ and $2 \mathrm{~mm}$ mesh sizes were selected for grid independence verification. imulation verification conditions: pipe diameter $\mathrm{D}=24 \mathrm{~mm}$, gas Reynolds number $\mathrm{Re}$ is 20000 , twist rate of twisted band is 6.2 , initial concentration of particles is $2 \%$.As shown in Fig. 5, the velocity distribution on the cross-section at $\mathrm{Z}=5 \mathrm{D}$ of the pipeline is selected for comparison. Under these three mesh size conditions, the velocity distribution curves obtained by the simulation are generally similar. However, compared with $2 \mathrm{~mm}$ (6541200cells) and 4mm (4209480cells), the mesh size of $8 \mathrm{~mm}$ ( 2865700 cells) varies greatly, and there a re fewermeshes near the wall, so the calculation accuracy is not enough. On the other hand, the grid size of $2 \mathrm{~mm}$ (6541200cells) and $4 \mathrm{~mm}$ (4209480cells) is basically the same, and the accuracy is not improved much near the wall. In order to improve the computing efficie ncy, the grid size of $4 \mathrm{~mm}$ (4209480cells) is finally selected as the computational grid. 


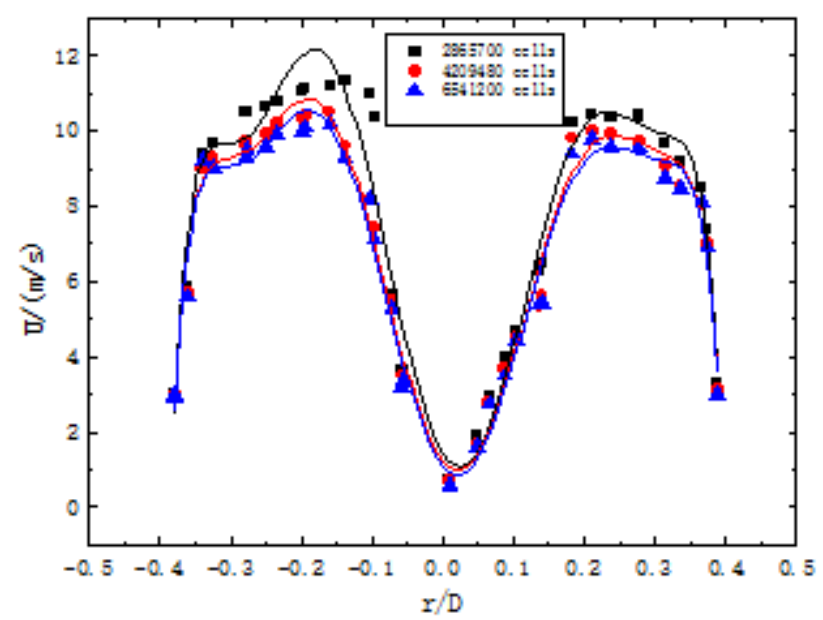

Figure 5. The velocity distribution on pipeline section at $Z=5 D$ with different mesh sizes

\subsubsection{Experimental verification of gas-solid two-phase spiral flow and heat transfer}

The numerical simulation results of gas-solid two-phase spiral flow a re compared with the experimental results to verify the feasibility. Contrast diagram as shown in figure 6 and 7 are the results of the validation, the pressure drop of the fluid $(\Delta \mathrm{P})$ and nusselt number $(\mathrm{Nu})$ with the Reynolds number $(\mathrm{Re})$ changes in gas -solid two phase flow. The pipe is $1.2 \mathrm{~m}$ long, $24 \mathrm{~mm}$ in diameter and the particle size is about $0.02 \mathrm{~mm}$. The concentration of solid particles used in the simulation is the ratio of solid volume to gas volume. From the simulation and experimental verification results, the error is very small, so the gas-solid two-phase flow in the gas hydrate pipeline can be calculated by numerical simulation method.

The Reynolds number is calculated as follows:

$$
\mathrm{Re}=\frac{\rho v d}{\mu}
$$

Where, $\mathrm{d}$ is the diameter of the pipe, $\mathrm{v}$ is the average velocity of particle flow, $\rho$ and $\mu$ are the density and dynamic viscosity of the gas, respectively.

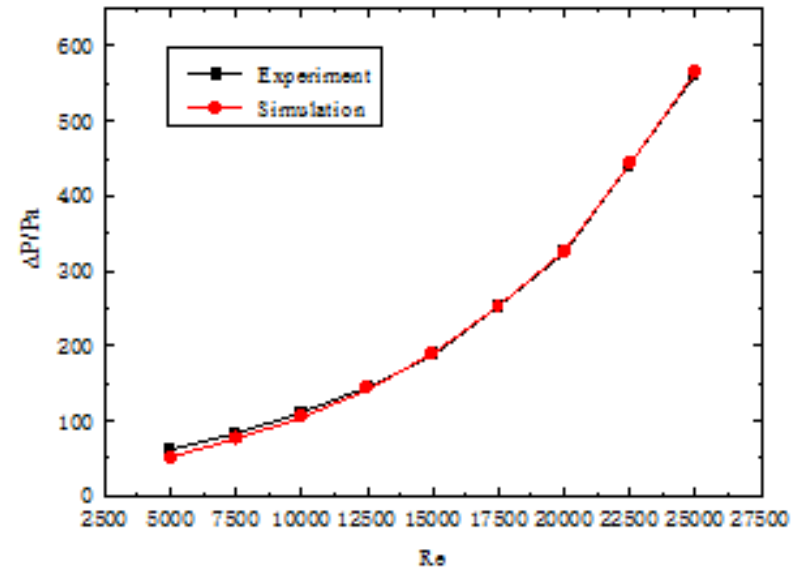

Figure 6. The effect of Reynolds number on pressure drop 


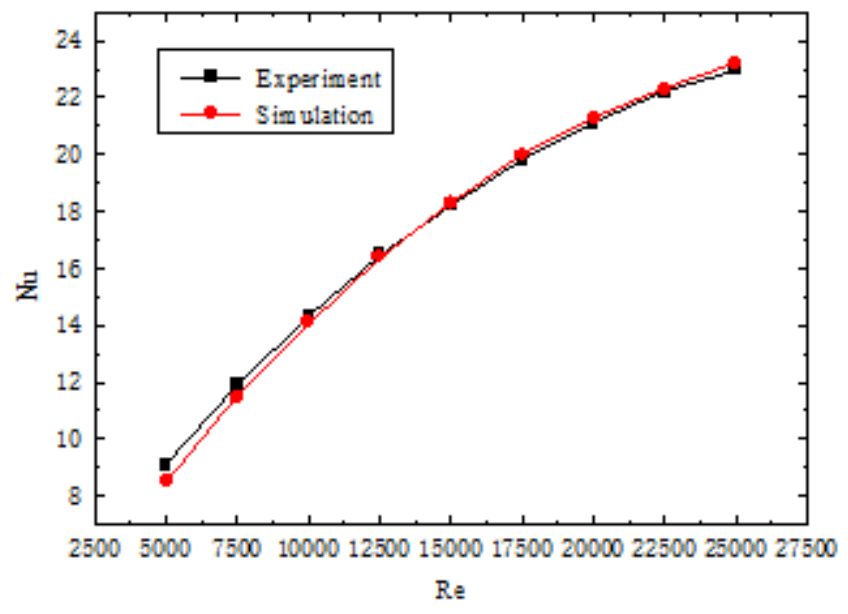

\section{Result Analysis}

Figure 7. The effect of Reynolds Number on Nusselt Number

The deposition and heat transfer of particles in spiral pipe flow were studied by using the short twisted band model. The velocity distribution, pressure drop distribution, particle deposition characteristics and heat transfer characteristics in the natural gas pipeline are mainly studied, and the effects of long twisted band and no twisted band as well as different Reynolds numbers are compared and analyzed.

\subsection{Velocity distribution law}

Fig. 8 shows the velocity distribution cloud map at each section under the condition of $\operatorname{Re}=15000$ and different torsional rates. It can be seen from the figure that in the light pipe without torsional band flat DC, the velocity is maximum in the central area of the pipe and then decreases uniform ly towa rds the pipe wall, and the velocity change at each section is not obvious. However, in the pipe into the twisted band, two distinct eddies are formed on both sides of the twisted band, with the maximum velocity at the center of the vortex. Along the direction of the pipe, the two vortices move from the near torsional band toward the wall. Compared with the short twisted band, the vortex always exists because the long twisted band is full of the whole pipe, while in the short twisted band pipe, the velocity tends to decrease as the particle transportation distance increases. And the lower the twist rate of the twisted band, the greater the velocity of the two vortex centers.
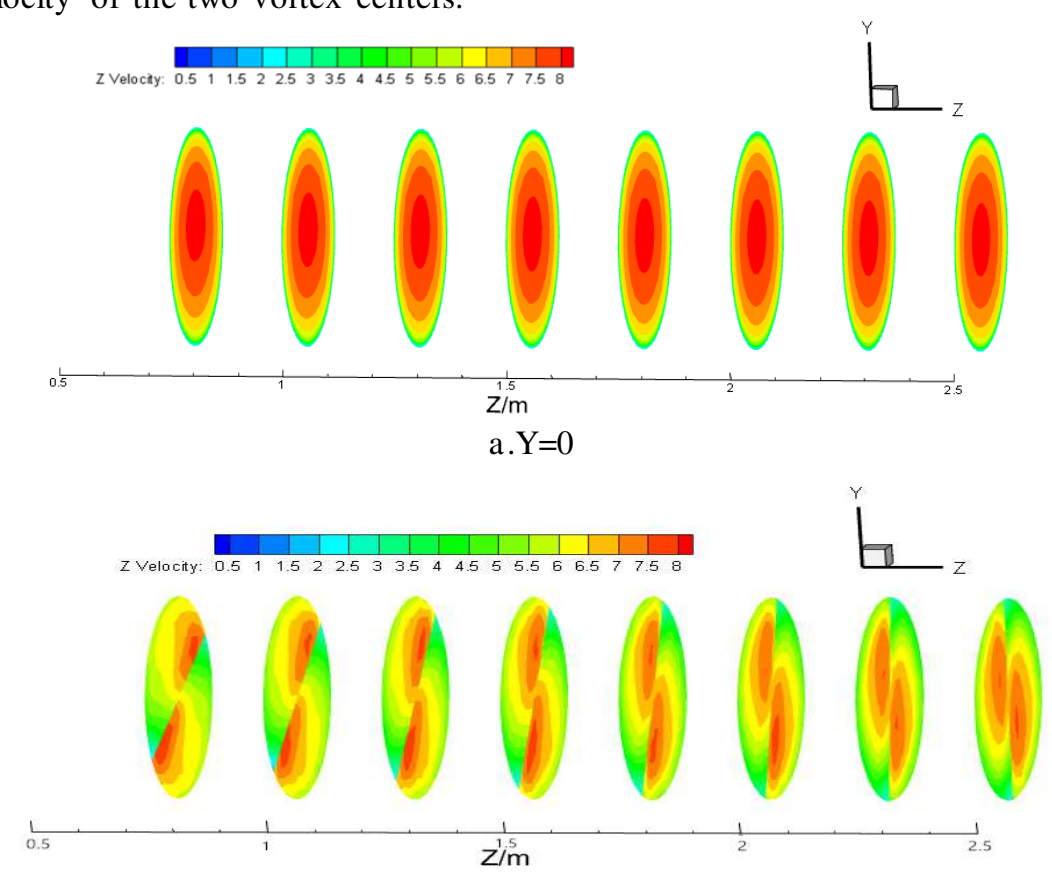

b. $Y=6.2$ 


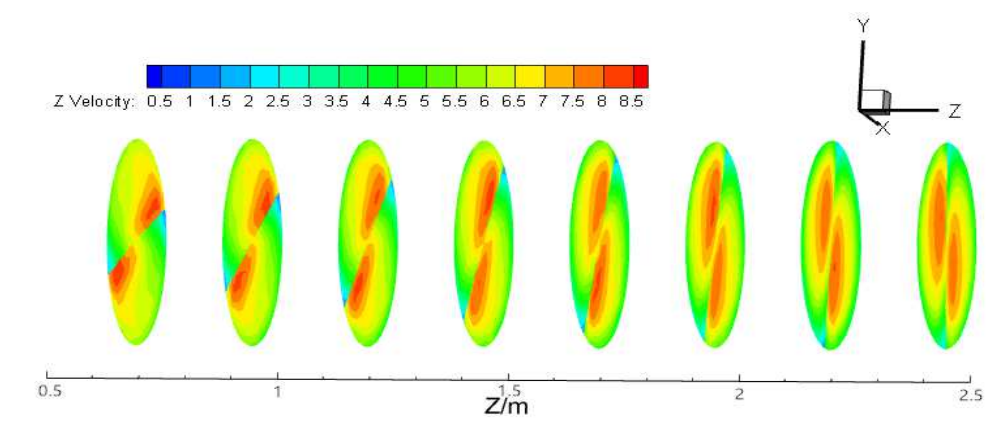

c. $Y=7.4$

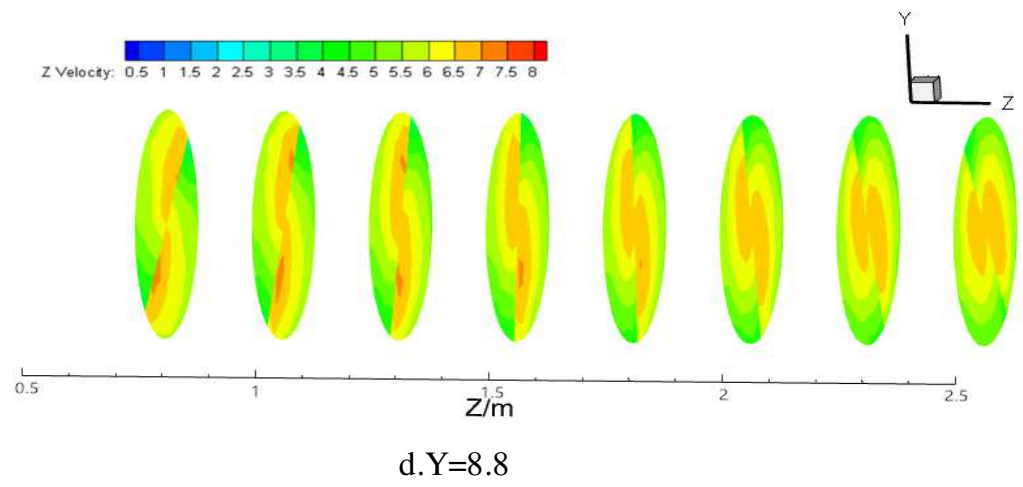

Figure 8. Velocity distribution clouds at different sections under $\mathrm{Re}=15000$ and different torsion rates

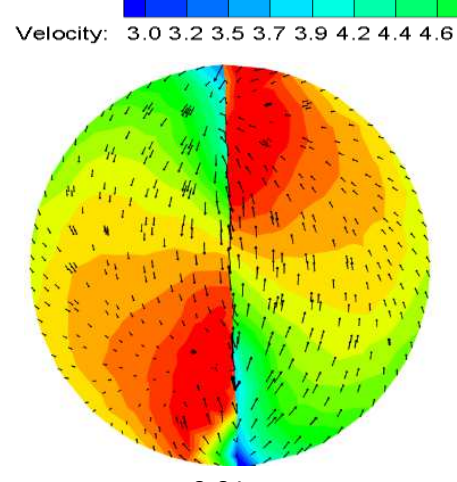

Figure 9. Velocity and vector distribution nephogram in the long twisted band $\mathrm{Y}=6.2$

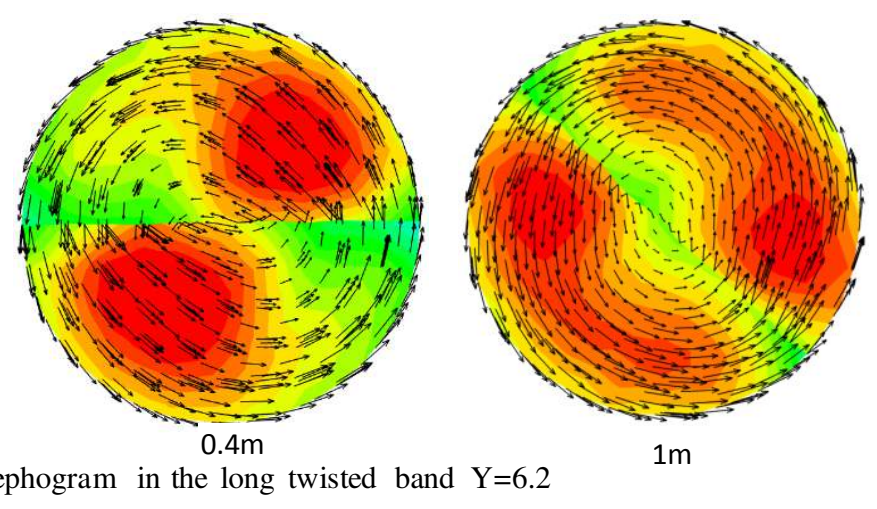

Fig. 9 shows the velocity and vector graphs at different sections. After the velocity distribution is stabilized, the velocity center can be obviously observed at the far end of the pressure surface of the pipeline, and there is an obvious wake. It can be seen from the vector line that the tangential velocity first increases and then stabilizes. The tangential velocity hardly attenuates in the process of hydrate transportation. And the tangential velocity value near the wall is the largest. It is because of the tangential velocity that hydrate particles larger tangential force with the wall, make the hydrate particle is not easy to deposit, tangential velocity at the same time spin in the hydrate particles themselves, make hydrate particle is not easy to reunite and deposition, since the long twisted band rotates all the way, the constant tangential velocity can further expand the safe flow boundary of hydrate.

\subsection{Distribution law of spiral flow pressure drop of pipeline}

\subsubsection{Influence of Reynolds number on pressure drop}


Fig. 10 shows the relationship between pipe pressure drop and Reynolds number when a twisted band with a torsion rate of 6.2 is placed. It can be seen from the figure that at the same section of the pipe, the pressure drop of the pipe increases with the increase of Reynolds number, and the higher the Reynolds number is, the more the pressure drop increases. Along the direction of the pipe, the pressure decreases more obviously with the increase of the particle transportation distance, and the slope of the curve in the figure is larger and larger, which is similar to a parabola.

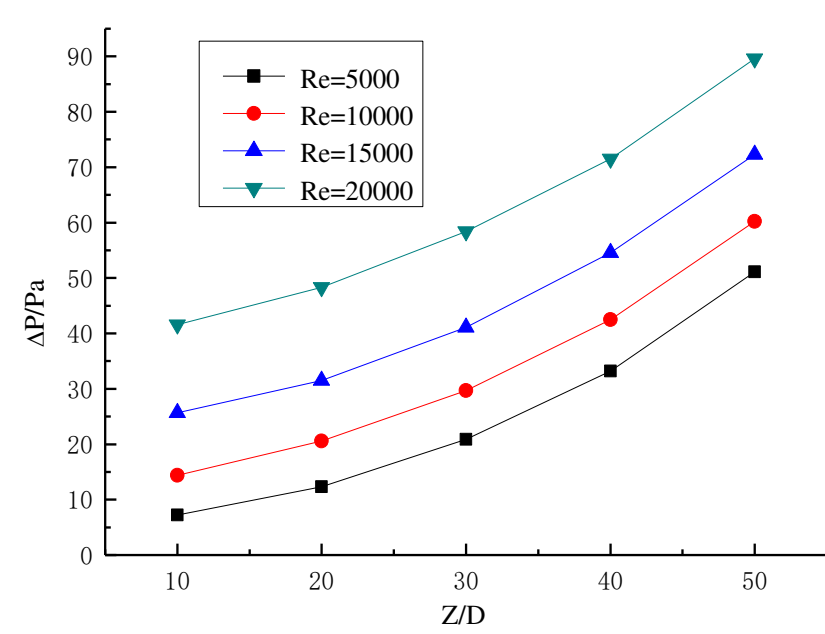

Figure 10. Relationship between Reynolds number and

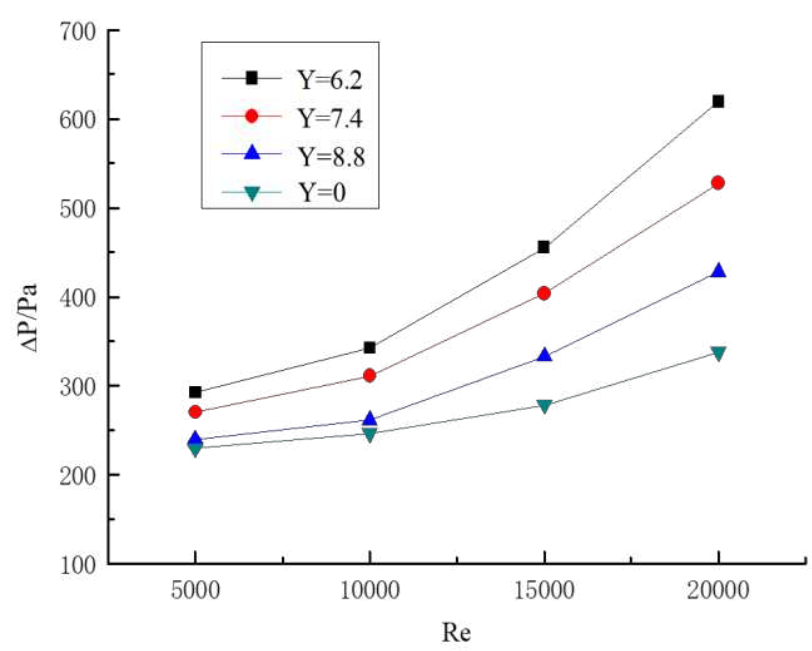

Figure 11. Relationship between twist rate and pressure drop

\subsubsection{Influence of torsion ratio on pressure drop}

Fig. 11 shows the relationship between Reynolds number and pressure drop under different torsional rates in horizontal pipelines. It can be seen from the figure that the pressure drop is affected by the twist rate. The smaller the twist rate is, the greater the pressure drop of the pipeline is. When a twisted band is placed in the pipe, the pressure drop will change significantly. The reasons are analyzed as follows: compared with the straight light pipe flow, the torsional belt placed in the pipe produces resistance to the fluid, and the spiral flow generated by the torsional belt disturbs the boundary layer of the pipe and causes turbulent flow. As a result, the pressure drop of the torsional belt placed in the pipe is higher than the pipe without torsional belt.

In the case of constant particle Reynolds number, the greater the torsion ratio of the twisted band placed in the pipe, the weaker the spiral flow strength, the smaller the tangential velocity of the spiral flow and the smaller the axial velocity loss, and the smaller the increase in pressure drop of the pipe.

\subsection{Analysis of heat transfer law}

\subsubsection{Axial temperature distribution rule}

Fig. 12 shows the temperature distribution cloud map at each section of the pipe with $\mathrm{Re}=20000$ and different torsion rates. As can be seen from the figure, the temperature distribution in the pipe without torsional bands is relatively uniform and layered at the beginning. Along the direction of the pipe, due to the effect of heat transfer, the temperature from the center of the pipe to the wall decreases gradually, and the temperature in the center of the pipe is the highest. After adding the twisted band, two vortices will be formed on both sides of the twisted band, and the temperature at the two vortex center is the highest and gradually decreases from the center to the pipe wall. With the increase of the torsional band twist rate, the temperature in the vortex center is relatively smaller, because the smaller the twist rate, the stronger the spiral flow generated is, the stronger the boundary layer disturbance of the pipeline is, which is conducive to fluid heat transfer. Compared with the short twisted band before, the temperature of the whole pipe drops slowly. 


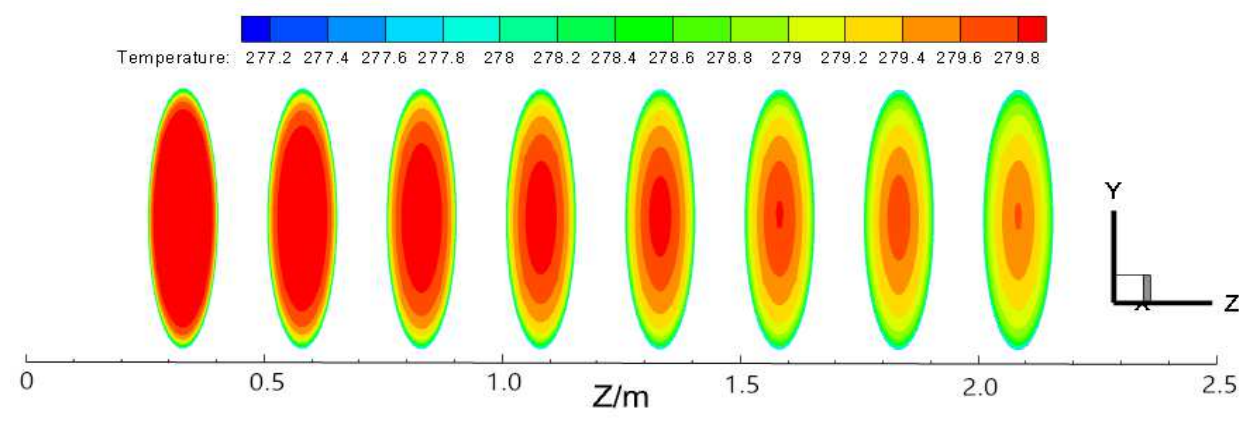

a. $\mathrm{Y}=0$

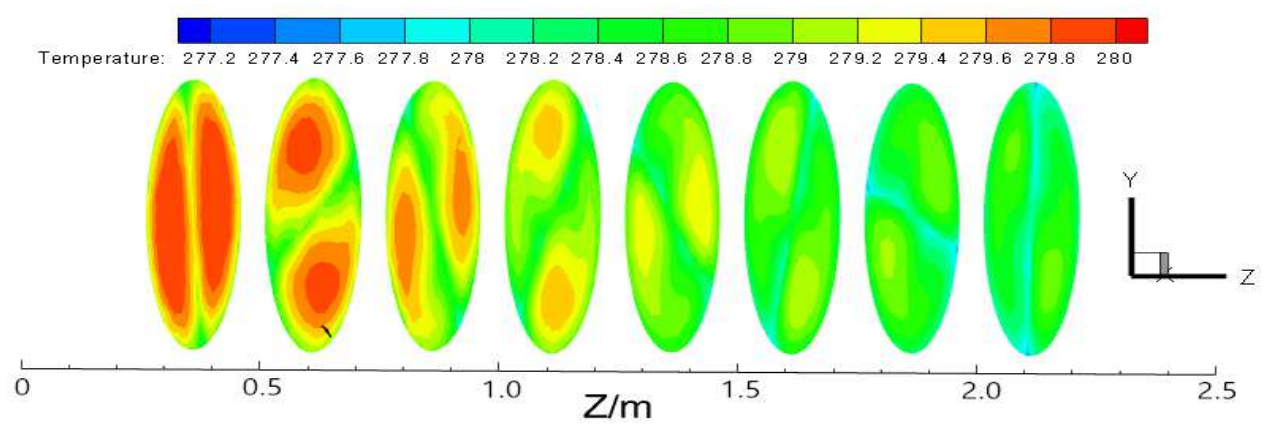

b. $Y=6.2$

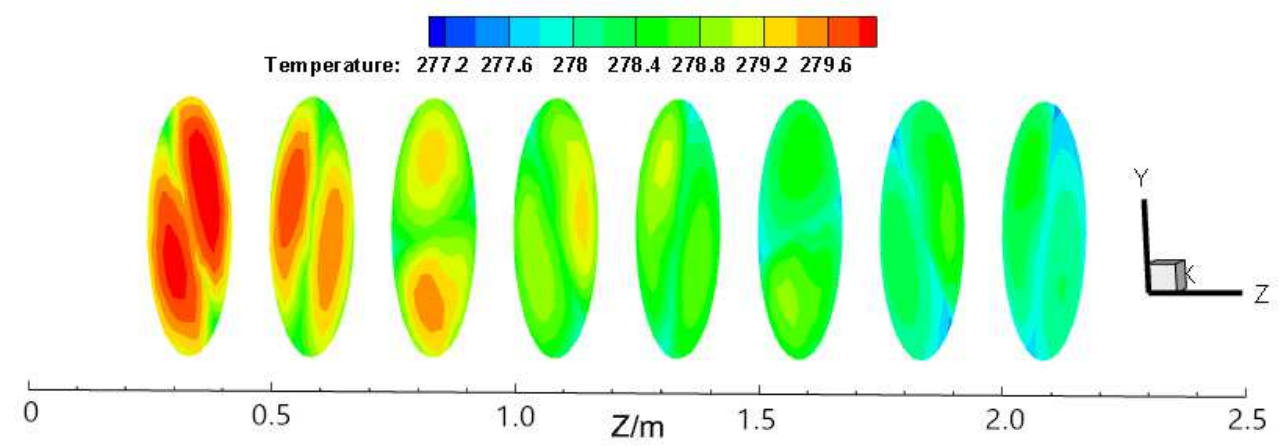

c. $Y=7.4$

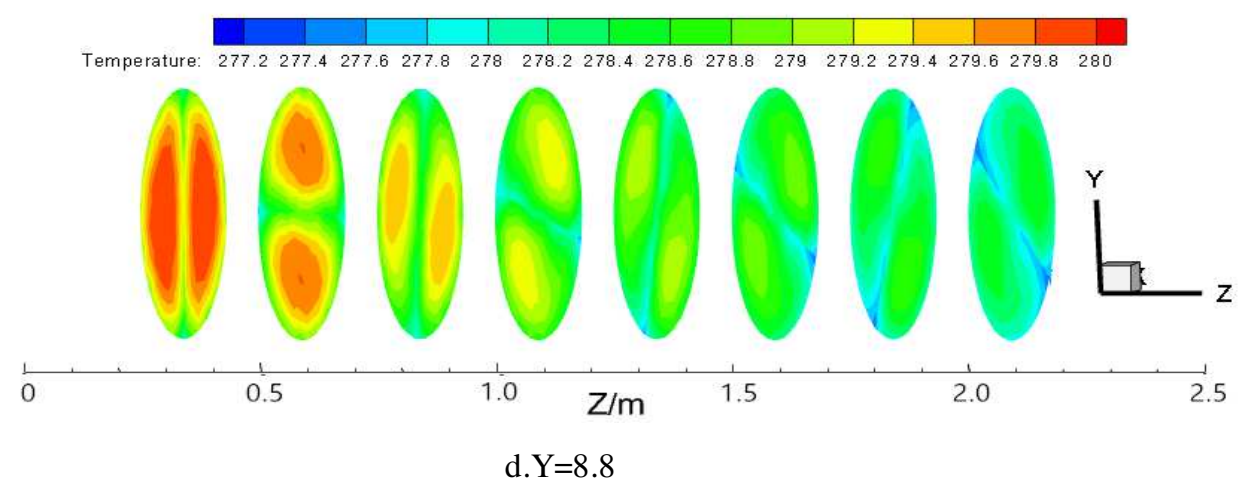

Figure 12. Temperature nephogram of each section in long twisted band pipeline with different twist ratio at $\mathrm{Re}=20000$ 3.3.2 Axial distribution law of Nussel number

Fig. 13 shows the change curve of the avera ge Nussel number Nu with Re in the long torsional pipe. In the plain DC of the light pipe without twisted band, Nu changes little with Re and approximates a horizontal line when Re is very small. A long torsional belt is placed in the pipeline, and when the fluid flows through the internal torsional belt, it will rotate, which enhances the tangential velocity of the fluid and continuously scour the boundary layer of the pipeline. Meanwhile, the disturbance of the boundary layer is a lso strengthened, and the turbulence intensity of the 
fluid near the wall is improved. The tangential motion of the fluid makes the boundary layer fluid and the main body mix and strengthen, thus effectively enhancing the convective heat transfer of the fluid. As you can see from the figure, the smaller the twist rate is, the larger $\mathrm{Nu}$ is. Because the lower the twist rate, the more twisted the twist, the longer the flow. In the case of the same mass flow rate, the longer the path of the fluid flow is, the larger the spiral flow formation and velocity is, and the greater the turbulence intensity of the fluid is, thus enhancing the heat transfer. Fig. 14 shows the $\mathrm{Nu}$ distribution curve of all sections under the condition of different torsion rates when $\mathrm{Re}=20000$. As can be seen from the figure, $\mathrm{Nu}$ of the straight light pipe flow is almost unchanged along the pipe. When the twisted tape is placed in the pipe, the Nusser number first strengthens and then decreases after about $20 \mathrm{D}$. Since the twisted band generates spiralflow at the beginning, which enhances the heat transfer of the pipe, Nu starts to become smaller with the heat exchange between the fluid and the pipe wall. Compared with the short twisted band, the long twisted band fills the whole pipe, so the spiral flow of the fluid in the pipe is maintained all the time, so the attenuation of the Nusser number is relatively small.

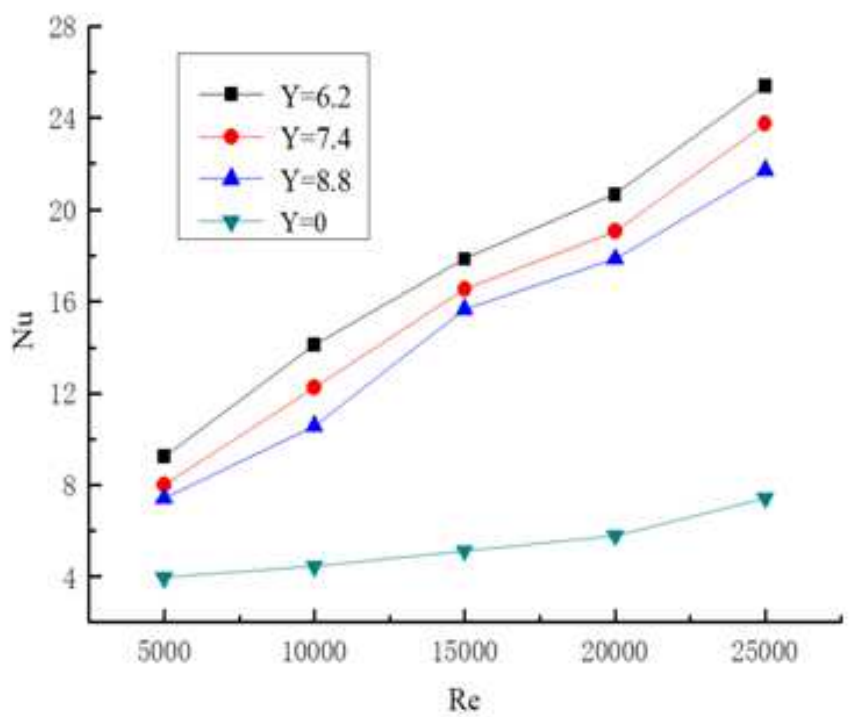

Figure 13. Variation curve of nus sel number with Re in long twisted band pipeline

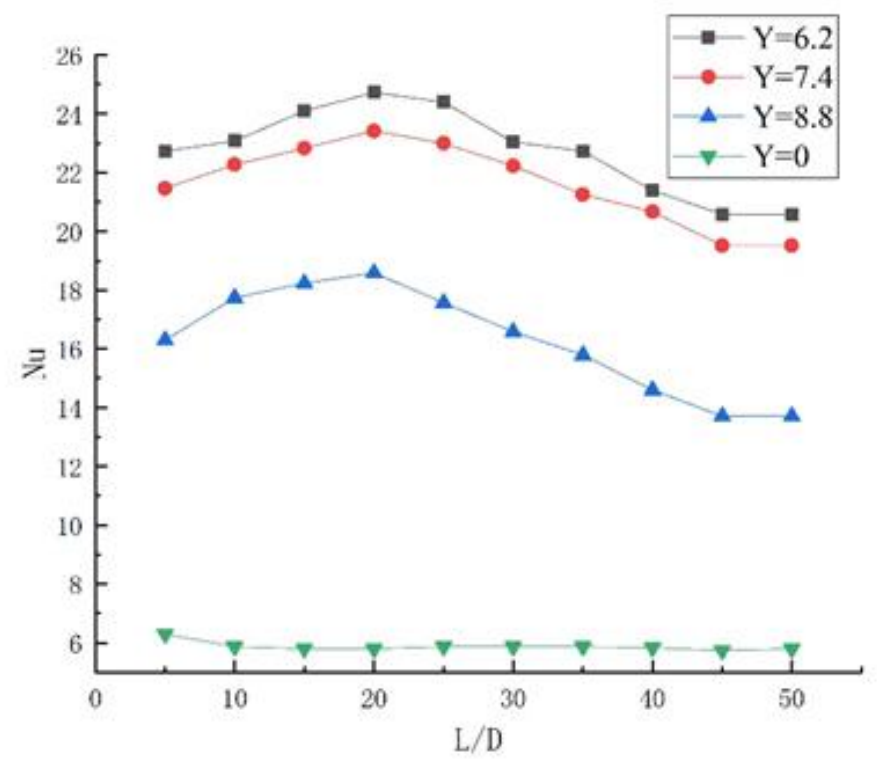

Figure 14. Nussel number distribution curves of different sections under different twist rates at $\mathrm{Re}=20000$

\subsubsection{Radial distribution law of Nussel number}

Fig. 15 shows the $\mathrm{Nu}$ distribution curve on the section $\mathrm{Z}=5 \mathrm{D}$ when $\mathrm{Re}=20000$. In a straight light pipe flow without torsion band, the Nusser number is in a parabolic shape with the opening downwards. At the center of the pipe, the Nusser number gradually decreases towards the pipe wall at the maximum, and at the near wall, the attenuation gradient of $\mathrm{Nu}$ is large. Because there is no swirl disturbance in the straight light pipe flow, the temperature at the 
center of the pipe is the highest, and the heat exchange temperature of the pipe wall decreases gradually. In the pipe with twisted tape, the curve of Nusserr number appeared a trough in the center of the pipe and a peak at half of the pipe diameter. Because of the presence of the twisted band, two eddies are formed on both sides of the twisted band to enhance the maximum heat transfer temperature of the fluid, which gradually attenuates to wards the tube wall and the twisted band. With the reduction of the twist rate, the Nussel number becomes larger and larger.

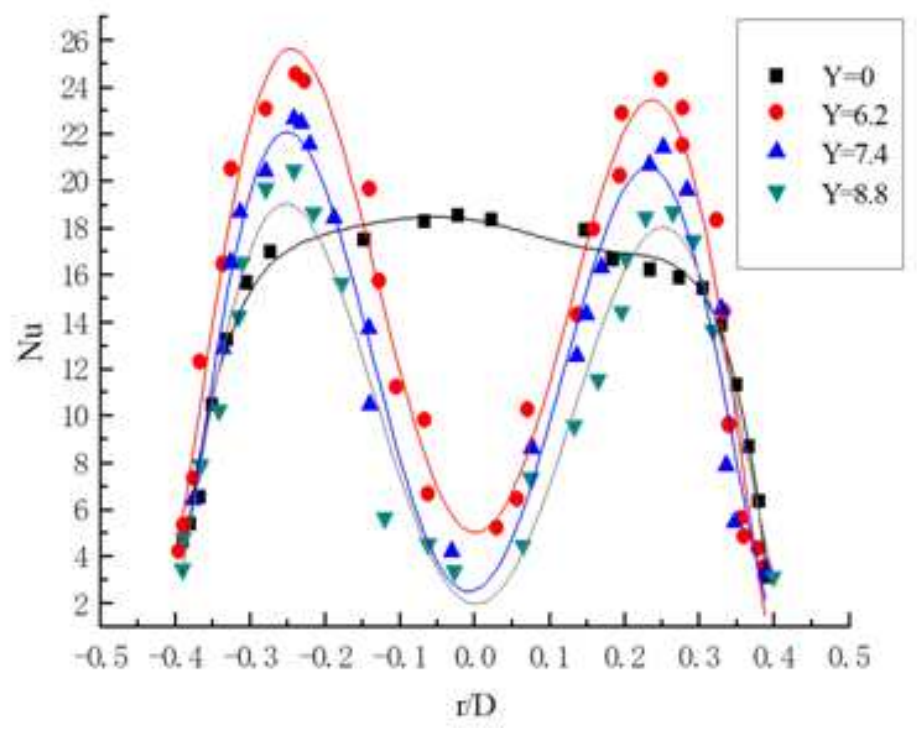

Figure 15. Nussel number distribution curve on $\mathrm{Z}=5 \mathrm{D}$ section at $\mathrm{Re}=20000$

\subsection{Particle sedimentation rule}

Fig. 16 shows the concentration distribution of hydrate particles at different positions. It can be seen that the concentration distribution of hydrate particles in the in itial section is relatively uniform when the long twisted band is used to spin. With the increase of distance, the concentration of hydrate particles presents an obvious symmetrical center distribution, and the hydrate particles are flung to the near pressure surface of the twisted band due to centrifugal force. At the same time, because it is located in the center of the spiral vortex, the pressure is low, forcing the surrounding particles to concentrate to the low pressure area. By comparing different twist rates, the smaller the twisted rates was the more obvious in the concentration area. In the absence of twisted bands, particles begin to deposit significantly at the position of $0.7 \mathrm{~m}$ and reach the maximum particle concentration at the position of $1.5 \mathrm{~m}$, indicating that this point is a possible location for blockage. When the spiral flow is not completely attenuated, the concentration of particles in each section is almost the same, and the concentration of particles in the pipe wall reaches the maximum and is evenly distributed. However, there are some obvious deposits at the $1.6 \mathrm{~m}$ position after the spiral flow attenuation. The transport distance has been increased by more than 2 times compared with the condition without cyclone. However, the concentration distribution in all sections remained a lmost the same during the whole spinning process, indicating that no obvious deposition of particles occurred.

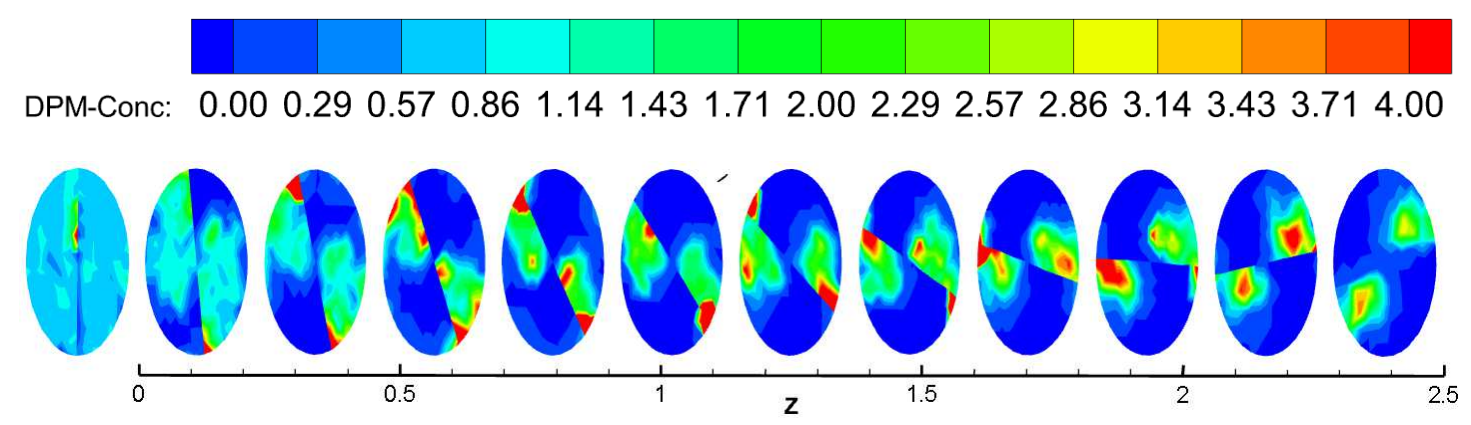

(a) Long twist band $\mathrm{Y}=6.2$ 


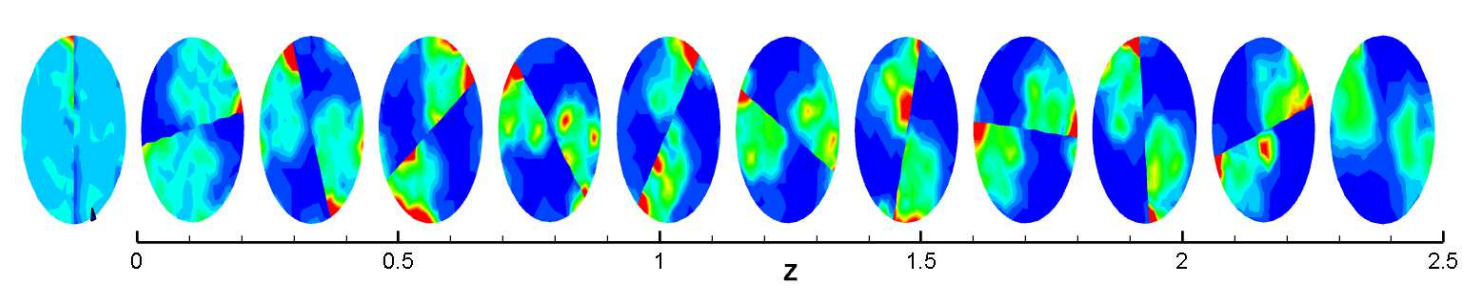

(b) Long twist band $\mathrm{Y}=7.4$

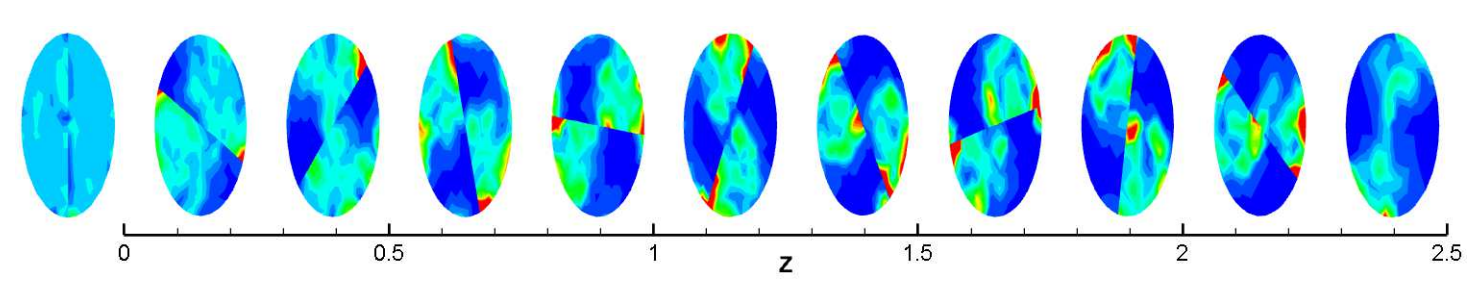

(c) Long twist band $\mathrm{Y}=8.8$

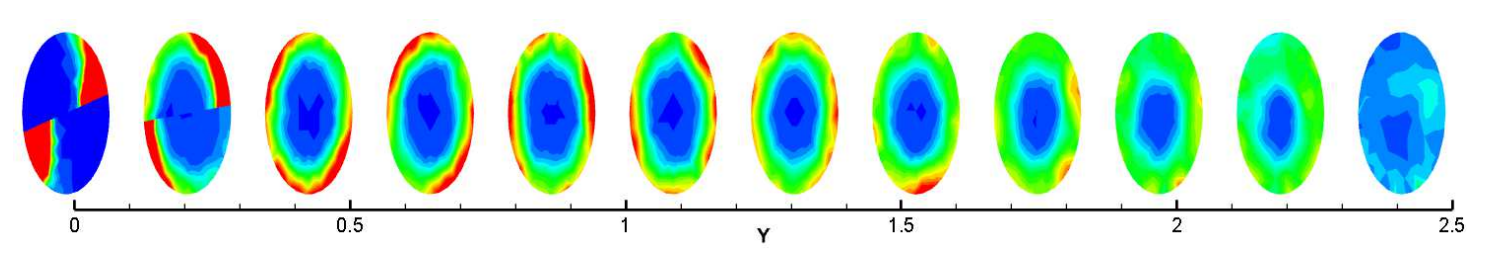

(d) Short twist band $Y=6.2$

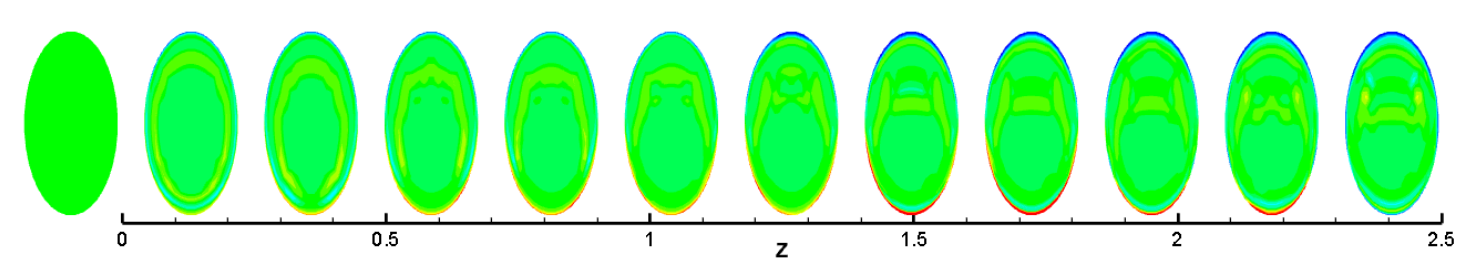

(e) No twist band

Figure 16. Distribution of particle concentration at different sections of the pipeline

Fig. 17 shows the distribution curve of the volume fraction of particles along the pipe when $\mathrm{Y}=6.2$ and $\alpha_{0}=1 \%$. The figure shows the volume fraction of particle deposition in the long torsional belt under three different Reynolds Numbers of $\mathrm{Re}=3000,5000$ and 10000 . When $\mathrm{Re}=3000$, the curve has a peak value at $\mathrm{Z}=56 \mathrm{D}$ approximately. The peak value represents the gradual accumulation of particles here and the point is considered as the deposition point of particles. With the increase of Reynolds number, when the curve tends to the horizontal line with no peak value at $\mathrm{Re}=5000$ and 10000, it can be considered that the particles a re not deposited in the pipe. The curve of $\mathrm{Re}=5000$ is slightly higher than that of $\operatorname{Re}=10000$, which indicates that the larger the Reynolds number is, the more difficult it is to deposit. The curve of $\mathrm{Re}=10000$ rises slightly because of the centrifugal motion of the particles in the long torsional band, some of the particles will stick to the wall of the tube and the tangential force of the spiral flow is not enough to make it fly a way. When $Y=6.2$ and initial concentration $\alpha_{0}=1 \%$, the criticaldeposition Reynolds number of particles is with in the range of 3000-5000.Fig. 18 shows the volume fraction distribution curve of particles along the pipeline when $Y=6.2$ and $\alpha_{0}=8 \%$.Compared with the figure above, the particles were deposited when $\operatorname{Re}=4000$, indicating that the critical deposition Reynolds number of the particles was $4000-5000$ when $\mathrm{Y}=6.2$ and initial concentration $\alpha_{0}=8 \%$. Because the higher the particle concentration is, the easier it is to deposit, and the higher the critical Reynolds number can be deposited. By comparing the two pictures, it can be concluded that the greater the particle concentration is, the closer the particle deposition is to the pipe inlet, and the more particles are deposited. 


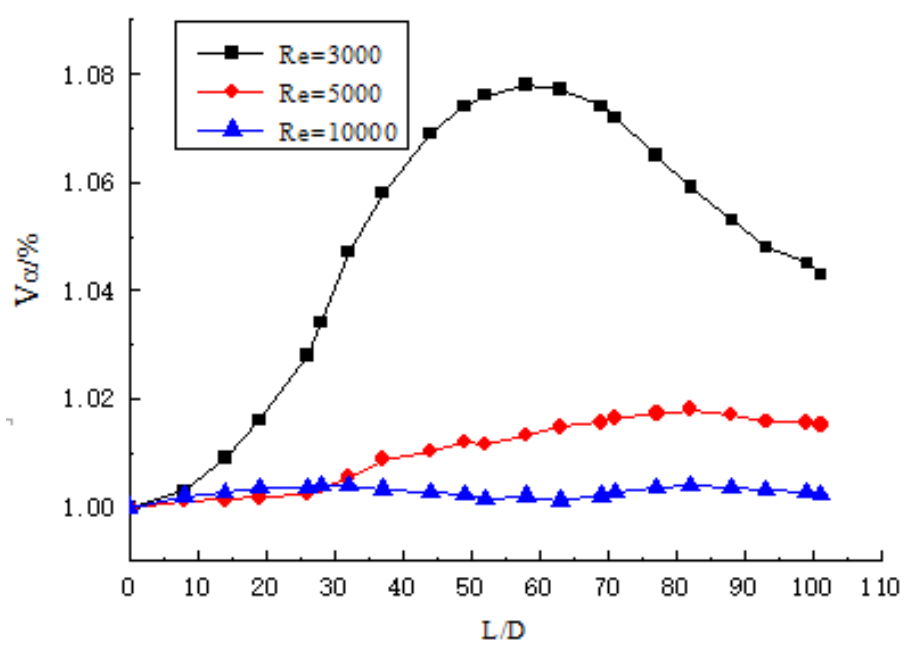

Figure 17. Volume fraction distribution curve of particles along pipeline at $\mathrm{Y}=6.2$

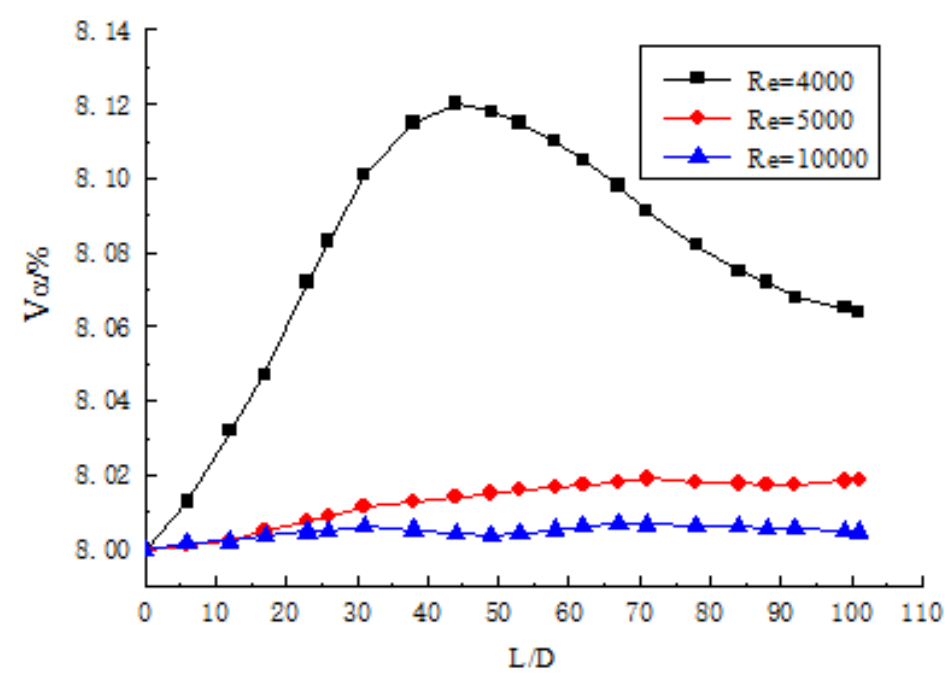

Figure 18. Volume fraction distribution curve of particles along pipeline at $Y=6.2, \alpha_{0}=8 \%$

Fig. 19 shows the distribution curve of volume fraction of different twisted band particles along the pipe when $\operatorname{Re}=4000$ and $\alpha_{0}=8 \%$. The twisted band with a twist rate of 6.2 short twisted band and long twisted band as well as flat light tube without twisted band were compared respectively, the curve in the figure shows an increasing trend. In the straight light tube flow without torsion band, the particles were deposited at $Z=12 \mathrm{D}$, where the volume fraction of the particles is the largest. In the short twisted band with $\mathrm{Y}=6.2$, particles were deposited at $\mathrm{Z}=28 \mathrm{D}$, and the peak value of the curve was smaller than that without twisted band. In the long twisted band with $Y=6.2$, the deposition location of particles is approximately at $Z=42 \mathrm{D}$, and the particles deposited in the pipeline are the least. It can be seen that the long twisted band has the best carrying effect, followed by the short twisted band, whose carry ing distance is about 3-4 times that of the non-twisted band. This result provides theoretical guidance for the safe transportation of hydrate. In Fig. 19, $\mathrm{Y}_{\mathrm{L}}$ represents the twist rate of the long twisted band, and $\mathrm{Y}_{\mathrm{S}}$ represents the twist rate of the short twisted band. 


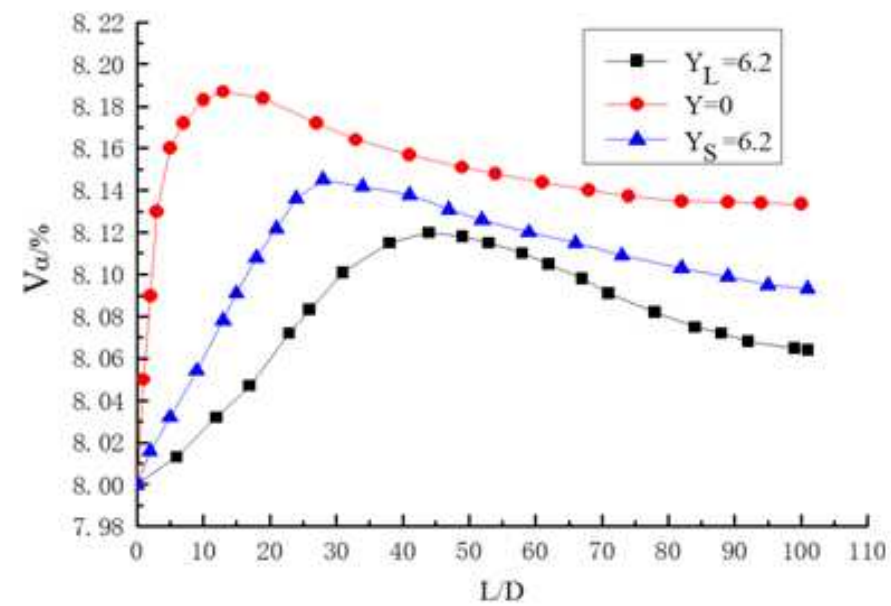

Figure 19. Volume fraction distribution curve of different twisted band particles along pipeline at $\operatorname{Re}=4000, \alpha_{0}=8 \%$

\section{Conclusions}

(1) In plain direct current of light pipe without torsional band, the velocity is maximum in the central area of the pipe and then decreases uniform ly towards the pipe wall, and the velocity change at each section is not obvious. However, in the pipe into the twisted band, two distinct eddies are formed on both sides of the twisted band, with the maximum velocity at the center of the vortex. Along the direction of the pipe, the two vortices move from the near torsional band toward the wall, it can effectively carry the hydrate particles deposited on the pipe wall.

(2) At the same section of the pipe, the pressure drop of the pipe increases with the increase of Reynolds number, and the higher the Reynolds number is, the more the pressure drop increases. Along the direction of the pipeline, the pressure drop becomes more obvious with the increase of particle transport distance, and the pressure drop curve is similar to a parabola. In the case of constant particle Reynolds number, the greater the torsion ratio of the twisted band placed in the pipe, the weaker the spiral flow strength, the smaller the tangential velocity of the spiral flow and the smaller the axial velocity loss, and the smaller the pressure drop of the pipe. Therefore, the pressure loss can be reduced as much as possible while the effect of spiral flow can be guaranteed.

(3) In a straight light pipe flow without torsion band, the Nusser number is in a parabolic shape with the opening down wards. At the center of the pipe, the Nusser number gradually decreases to wards the pipe wall at the maximum, and at the near wall, the attenuation gradient of $\mathrm{Nu}$ is large. In the pipe with twisted tape, the curve of Nusserr number appeared a trough in the center of the pipe and a peak at half of the pipe diameter. With the reduction of the twist rate, the Nussel number becomes larger and larger. Spiral flow can make the temperature distribution of the flow field in the pipeline more even and prevent the large number of formation of hydrate particles in the pipeline wall due to the large temperature difference.

(4) The larger the Reynolds number is, the less likely the particles are to deposit. The larger the particle concentration is, the closer the particle deposition is to the pipe inlet, more particle deposition. When the torsional rate of the long torsional band is 6.2 and the initial concentration of the particles is between $1 \%-8 \%$, the critical deposition Reynolds number of the particles is between $3000-5000$. Spiral flow has a good carry ing effect. Under the same working condition, the spiral flow carries hydrate particles at a distance about 3-4 times that of the flat and straight flow.

\section{References}

1 S. L. Wang, Y. C. Rao, Y. X. Wu, et al., Experimental Research on Gas-Liquid Two-Phase Spiral Flow in Horizontal Pipe. CHINA PET PROCESS PE14(3), 24-32, (2012).

2 S. L. Wang, Y. C. Rao, M. J. Wei, et al., Experimental Research on Law of Pressure Drop of Gas-liquid Two-phase Spiral Flow in the Horizontal Pipe. Sci. Tech. Engrg.13(3),659-663, (2013).

3 Y. Zhu, S. L. Wang and X. J. Shi, Numerical simulation of pipe spiral flow based on PHOENICS, CHINA PETROLEUM MACHINERY36(7) ,19-22, (2008).

4 M. Dou, S. F. Nan, P. Q. Duan, et al., Experimental Research on Anti-fouling of Heat Exchanger by Inserted Spring and Pulse Flow, J Chem Eng of Chinese Univ 24(5), 893-896, (2010).

5 L. L. Zhang and Y. Zhang, Flow field and flow characteristics along the spiral section of pipe, China Rural Water and Hydropower $9,48-50,(2011)$.

6 Y. C. Rao, Y. X. Zheng, S. L. Wang, et al., Numerical Simulation of Swirl Flow Rotated by the Vane in the Horizontal Pipe, Fluid Machinery44(4), 17-22, (2016). 
7 P. F. Zhao, W. C. Wang, Y. X. Li, et al., Numerical model of hydrate slurry flow in pipeline, Oil \& Gas Storage and Transportation35(3), 272-277,(2016).

8 G. C. Song, Y. X. Li, W. C. Wang, et al., Numerical simulation of pipeline hydrate slurry flow behavior based on population balance theory, Chem. Ind. Eng. Prog37(2), 561-568, (2018).

9 G. C. Song, Y. X. Li, W. C. Wang, et al., Numerical simulation of agglomeration process of hydrate particles in pipeline based on population balance theory, Petrochem Techno47(6), 573-582, (2018).

10 G. C. Song, Y. X. Li, W. C. Wang, et al. Numerical simulation of pipeline hydrate particle agglomeration based on population balance theory. Journal of Natural Gas Science and Engineering 51, 251-261, (2018).

11 G. C. Song, Y. X. Li, W. C. Wang, et al. Investigation on the mechanical properties and mechanical stabilities of pipewall hydrate deposition by modelling and numerical simulation. Chem. Eng. Sci192,477-487, (2018).

12 G. C. Song, Y. X. Li, W. C. Wang, et al. Numerical simulation of hydrate slurry flow behavior in oil-water systems based on hydrate agglomeration modelling. J. Petrol. Sci. Eng169,393-404, (2018).

13 J. Liang, Y. C. Rao, S. L. Wang, et al. Numerical Simulation of Spiral Flow and Heat Transfer in Natural Gas Hydrate Pipelines. Oil-Gasfield Surface Engineering37(2), 6-11, (2018).

14 J. Liang, Y. C. Rao, S. L. Wang, et al. Numerical Simulation of Spiral Flow and Heat Transfer of Hydrate Pipe Generated by Impeller, CHINA PETROLEUM MACHINERY46(2), 120-126, (2018).

15 J. Liang, Y. C. Rao, S. L. Wang, et al. Experimental Research of Solid Particle Transportation Based on Spiral Flow, Oil-Gasfield Surface Engineering 37(3), 1-5, (2018).

16 Y. Y. Cai, Y. C. Rao, S. L. Wang, et al., Numerical simulation of spiral transportof hy drate particles, Nat Gas Ind43(5), 66-73, (2018).

17 K. Chang, Y. C. Rao and S. L. Wang, Numerical Simulation of Spiral Flow of Gas Hydrate Particles in Gas Transmission Pipeline, CHINA PETROLEUM MACHINERY45(3), 107-113, (2017).

18 W. Y. Liu, J. Q. Hu, X. F. Li, et al., Assessment of hydrate blockage risk in long-distance natural gas transmission pipelines. J. Nat. Gas Sci. Eng60, 256-270, (2018).

19 E.P. Brown, D. Turner, G. Grasso, et al. Effect of wax/anti-a gglomerant interactions on hydrate depositing systems. Fuel264, 116573, (2020).

20 J. W. Nicholas, C. A. Koh, E. D. Sloan, et al., Measuring hydrate/ice deposition in a f low loop from dissolved water in live liquid condensate. AIChE J55(7), 1882-1888, (2009).

21 Lorenzo Di M, Aman Z M, Sanchenz Soto G, et al. Hydrate formation in gas-dominant system s using a single-pass flowloop. Energy Fuels28(5), 3043-3052, (2014).

\section{Acknowledgements}

This work is supported by the National Nature Science Foundation of China (No. 51574045\&51974037), Chan gzhou Applied Basic Research Project (No. CJ20200085)

\section{Author contributions}

Conceptualization, Yongchao Rao; methodology, Yongchao Rao and Lijun Li; software, Yongchao Rao and Lijun Li; validation, Yongchao Rao and Lijun Li; formal analysis, Yongchao Rao and Lijun Li; investigation, Yongchao Rao and Lijun Li; resources, Yongchao Rao, Lijun Li and Shuli Wang; data curation, Yongchao Rao, Lijun Li and Shuhua Zhao; writing - original draft preparation, Yongchao Rao and Lijun Li; writing - review and editing, Yongchao Rao; supervision, Yon gchao Rao; project administration, Yon gchao Rao; fund ing acquisition, Yon gchao Rao, Shuli Wang and Shidong Zhou; All authors have read and agreed to the published version of the manuscript.

\section{Competing interests}

The authors declare no competing interests. 
Figures

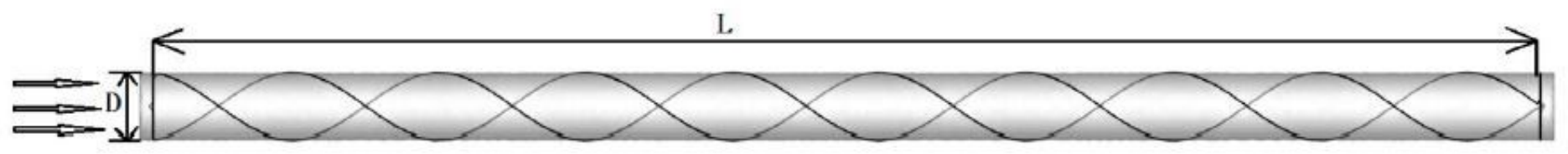

Figure 1

The physical model

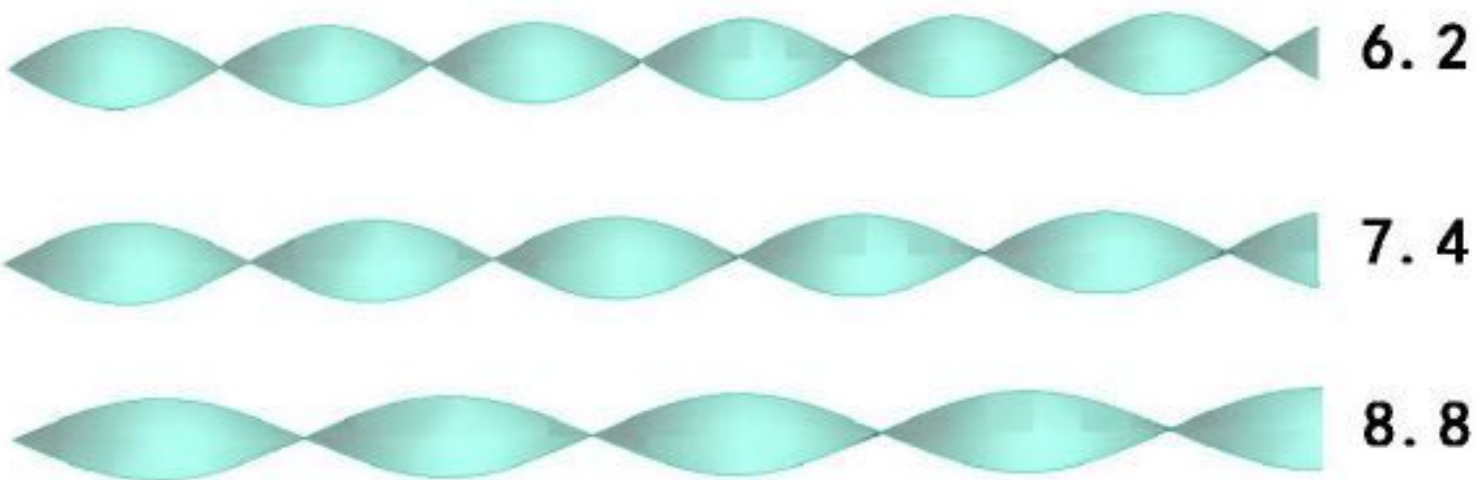

Figure 2

Twisted band model

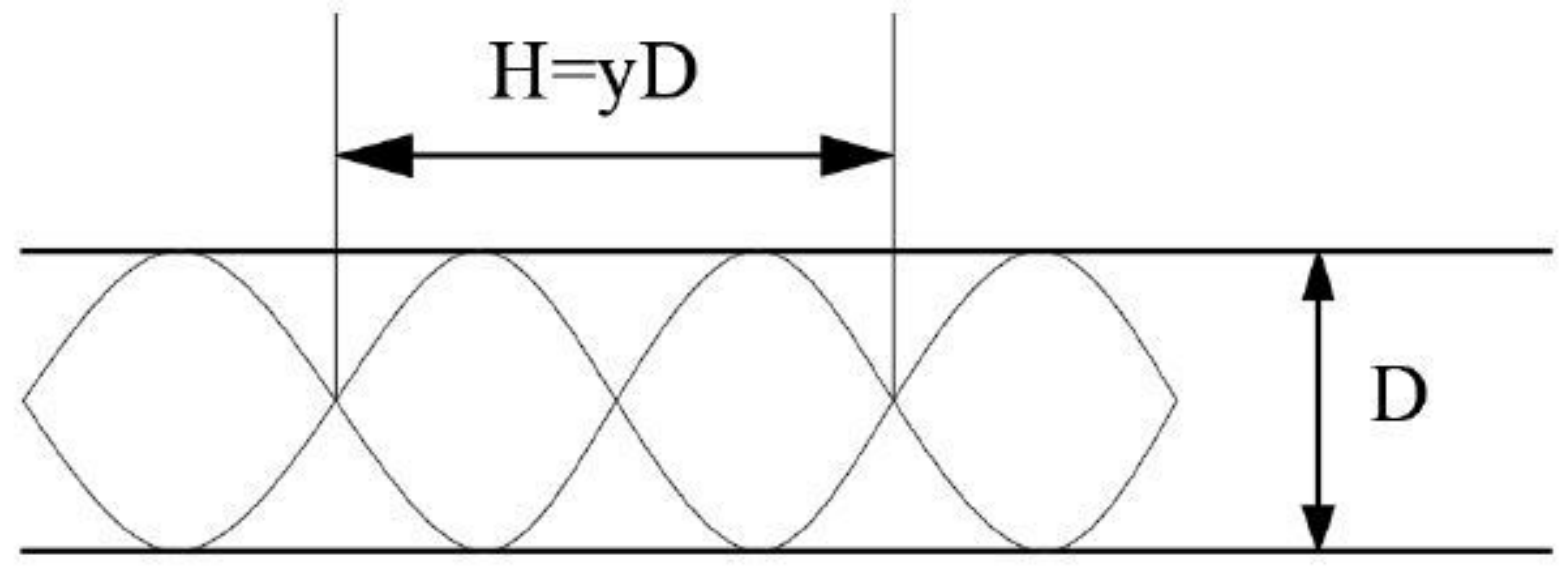

Figure 3

Twisted rate schematic diagram of twisted band 

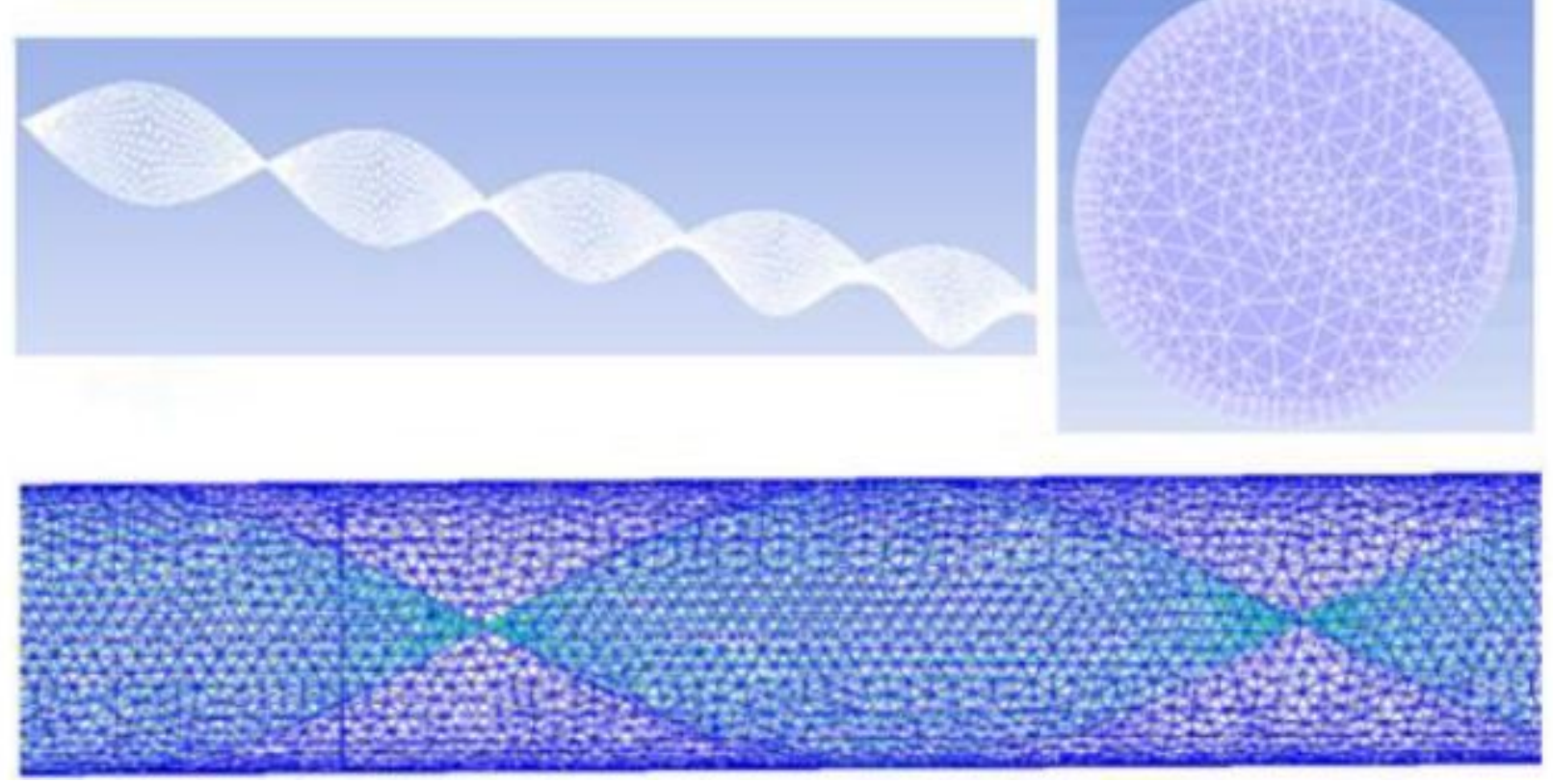

Figure 4

Calculation grid for long twisted band pipe section

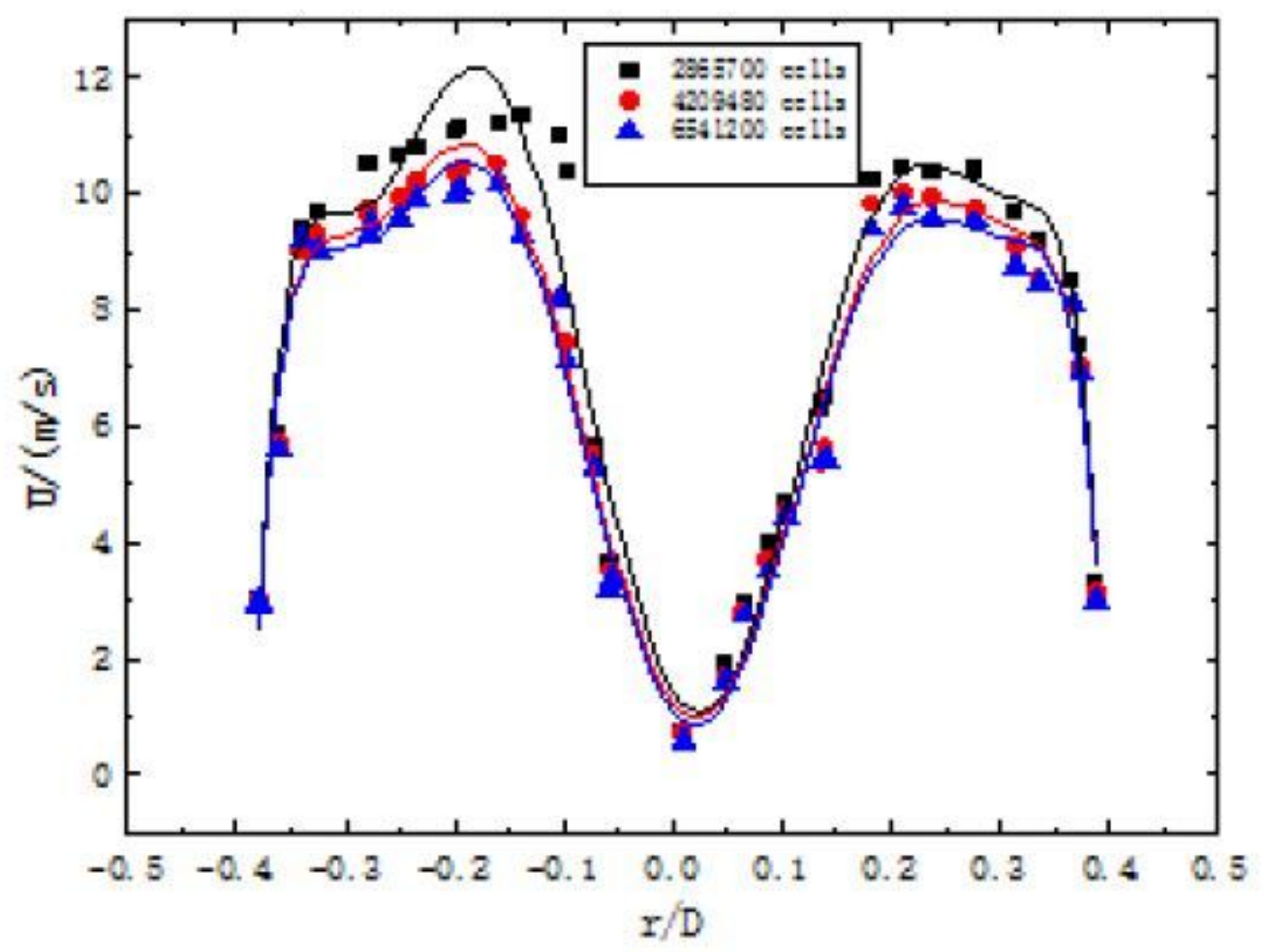

Figure 5

The velocity distribution on pipeline section at $Z=5 D$ with different mesh sizes 


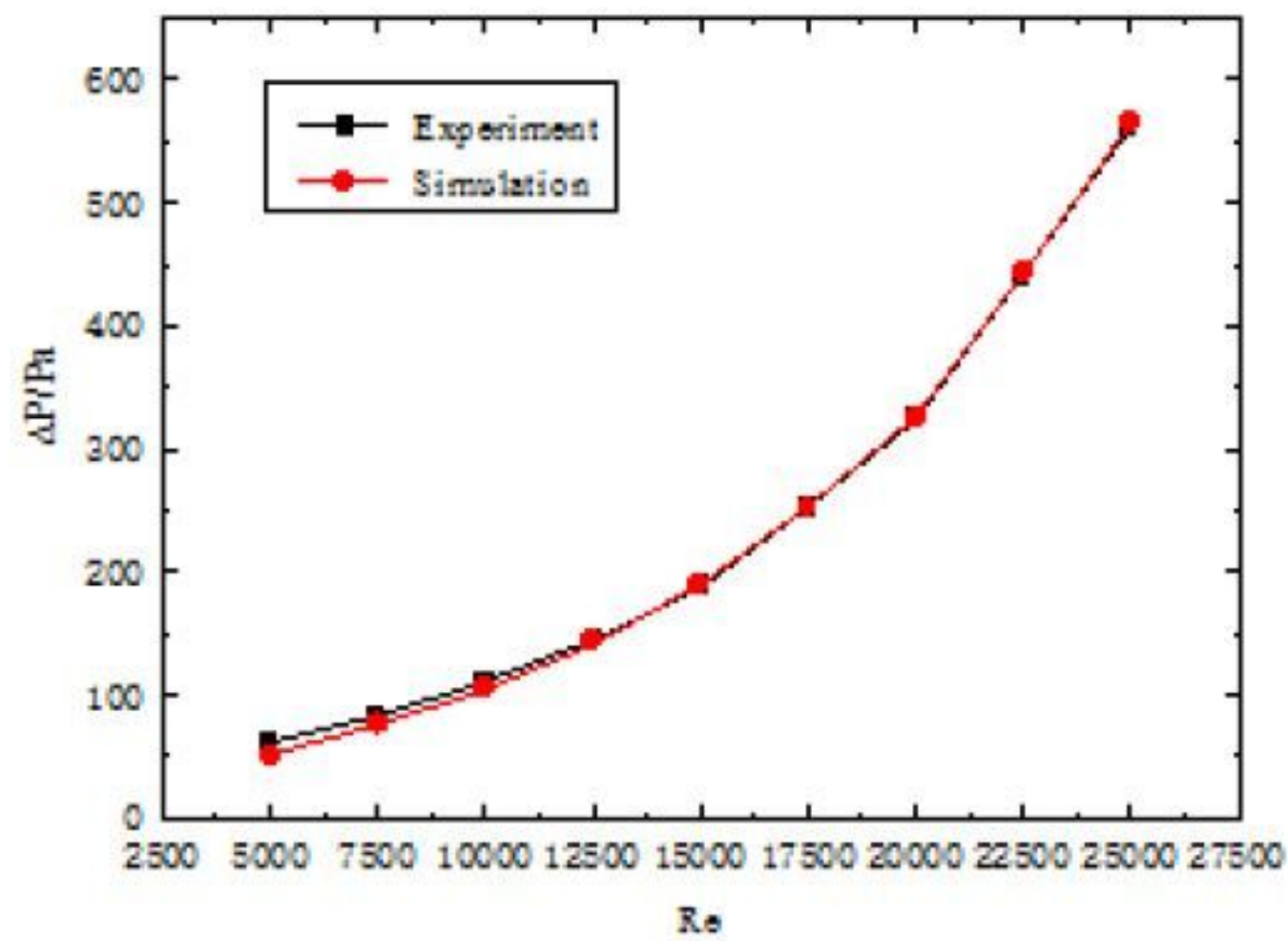

Figure 6

The effect of Reynolds number on pressure drop

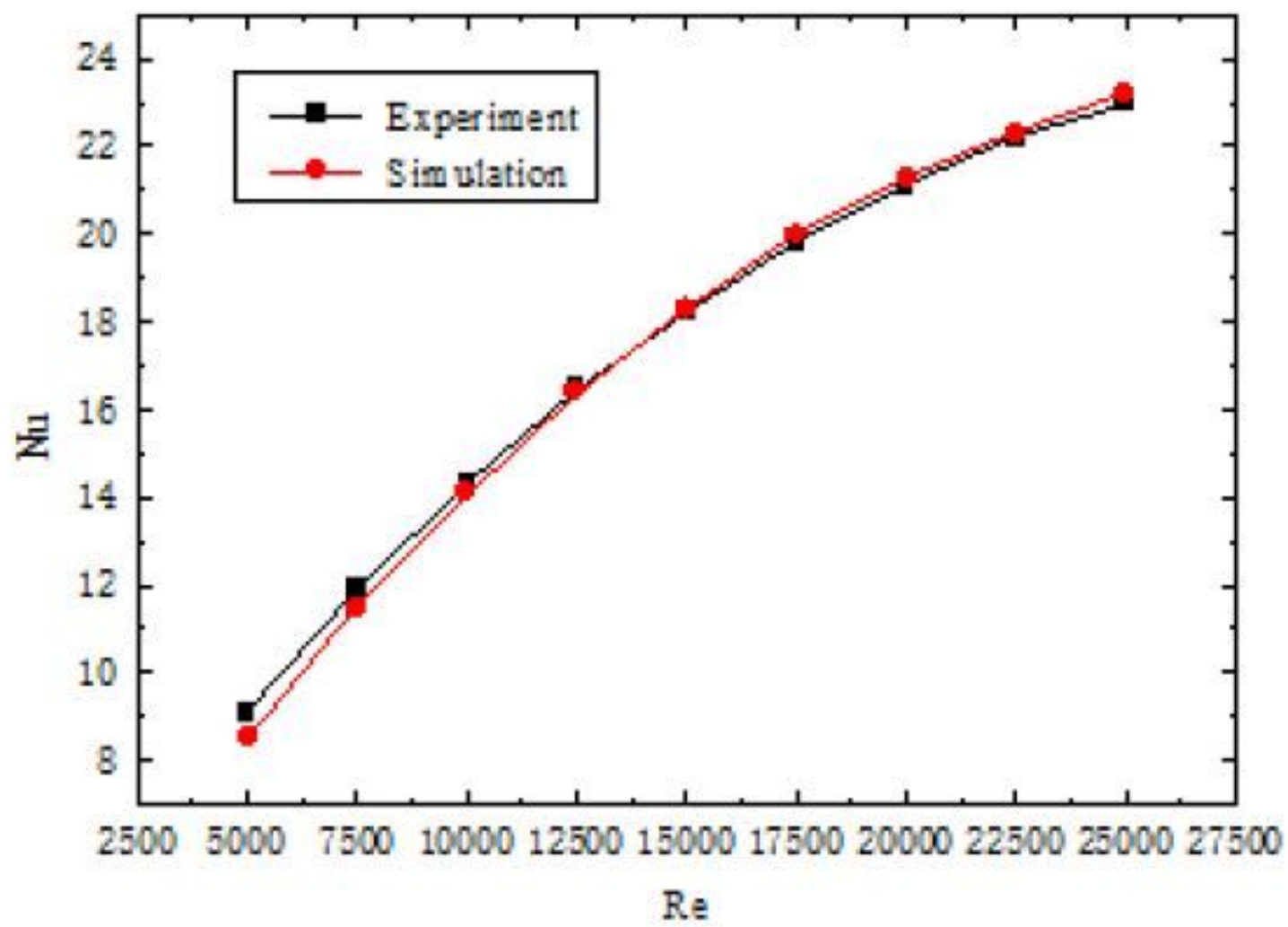

Figure 7 
The effect of Reynolds Number on Nusselt Number
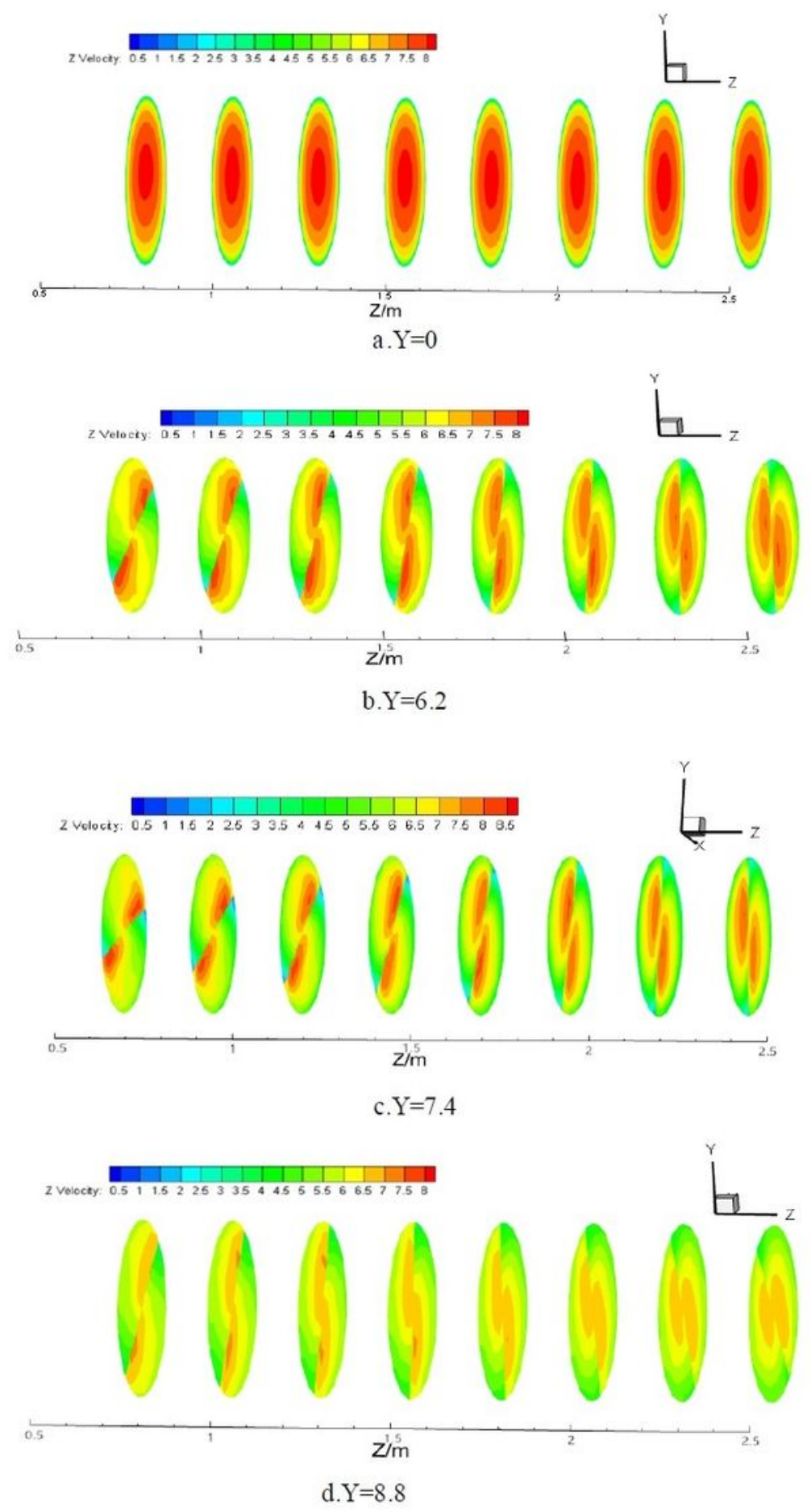

Figure 8

Velocity distribution clouds at different sections under $\mathrm{Re}=15000$ and different torsion rates 

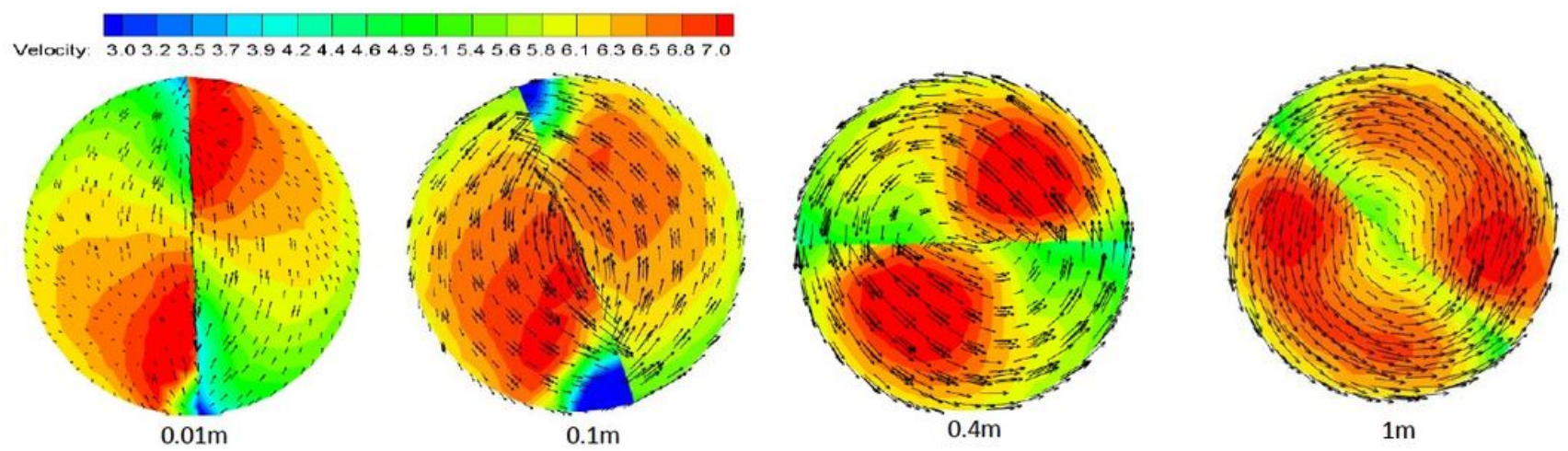

Figure 9

Velocity and vector distribution nephogram in the long twisted band $Y=6.2$

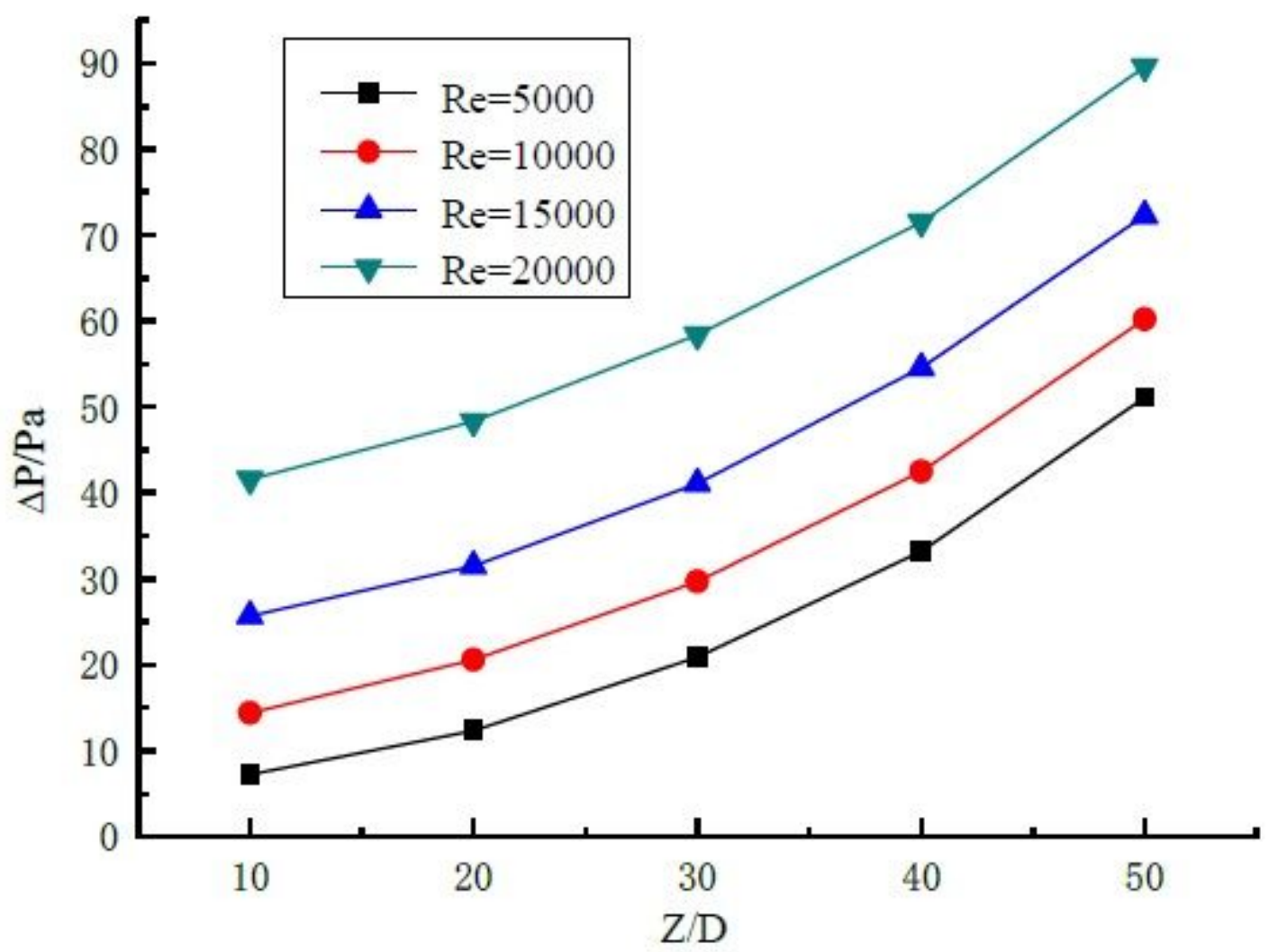

Figure 10

Relationship between Reynolds number and pressure drop 


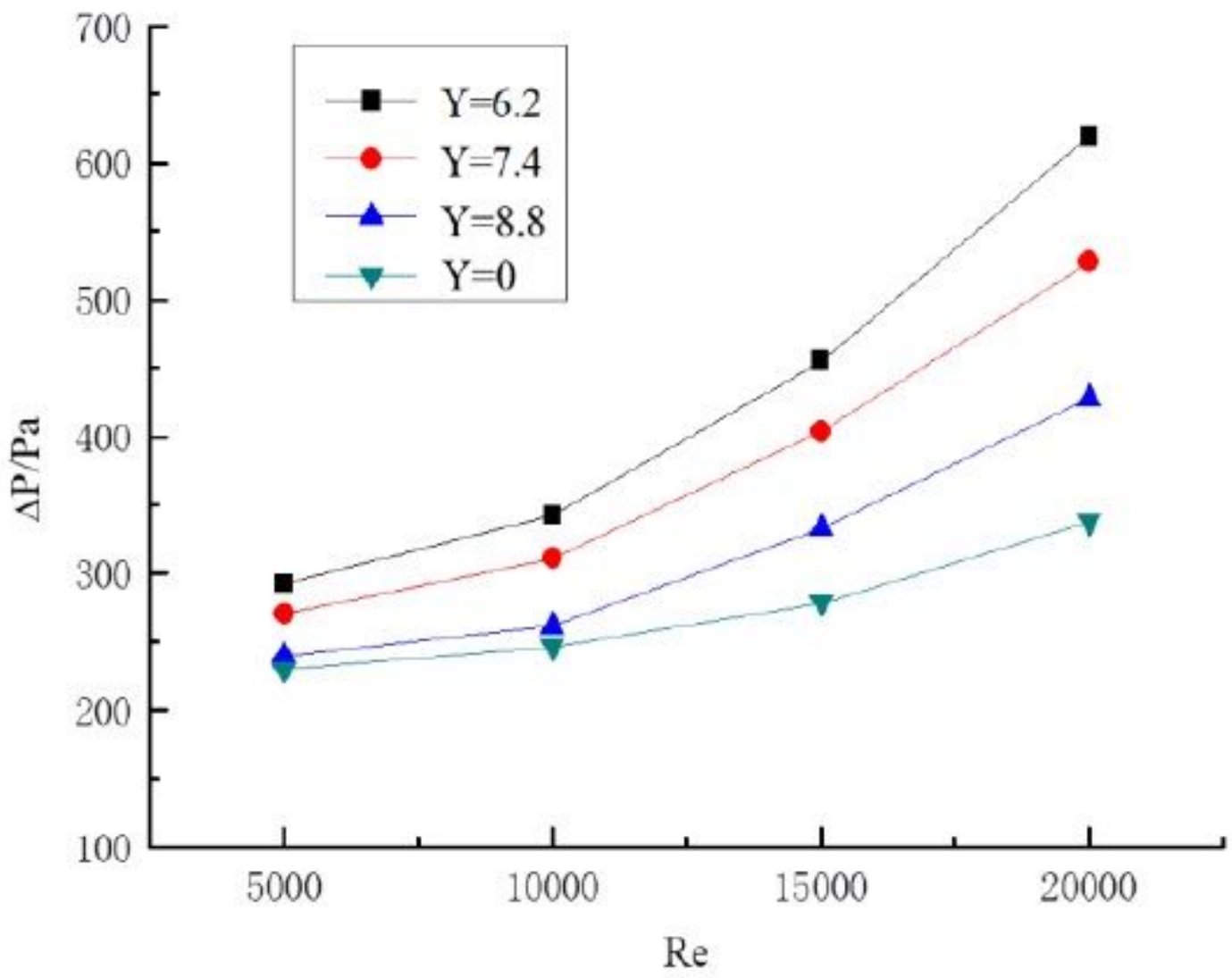

Figure 11

Relationship between twist rate and pressure drop 


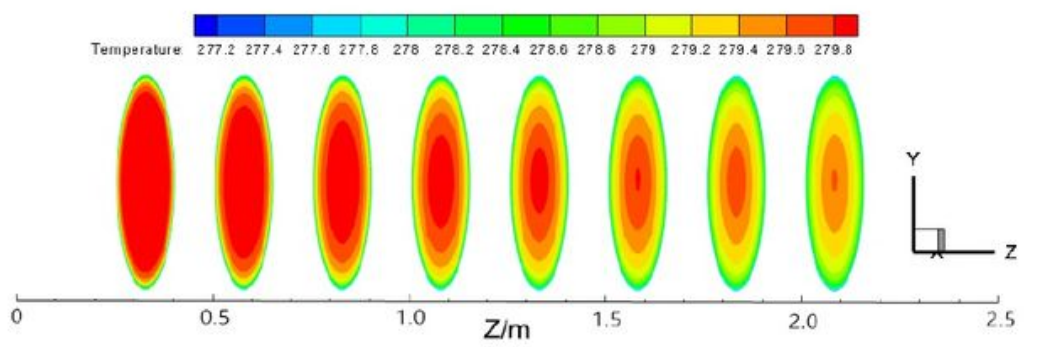

a. $\mathrm{Y}=0$

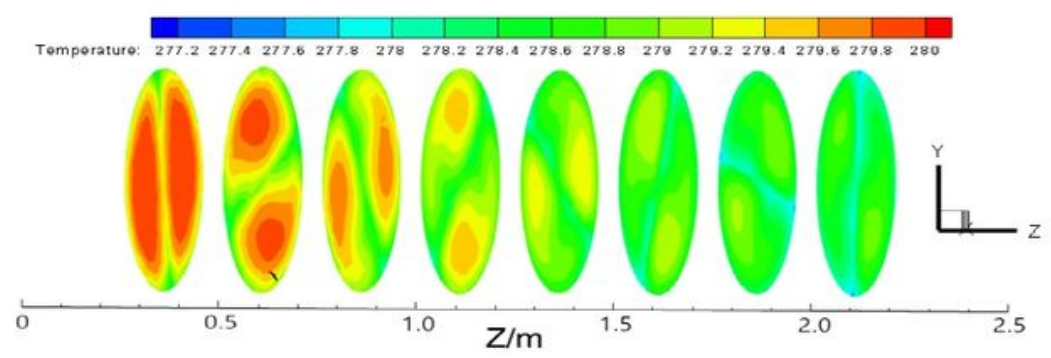

b. $\mathrm{Y}=6.2$
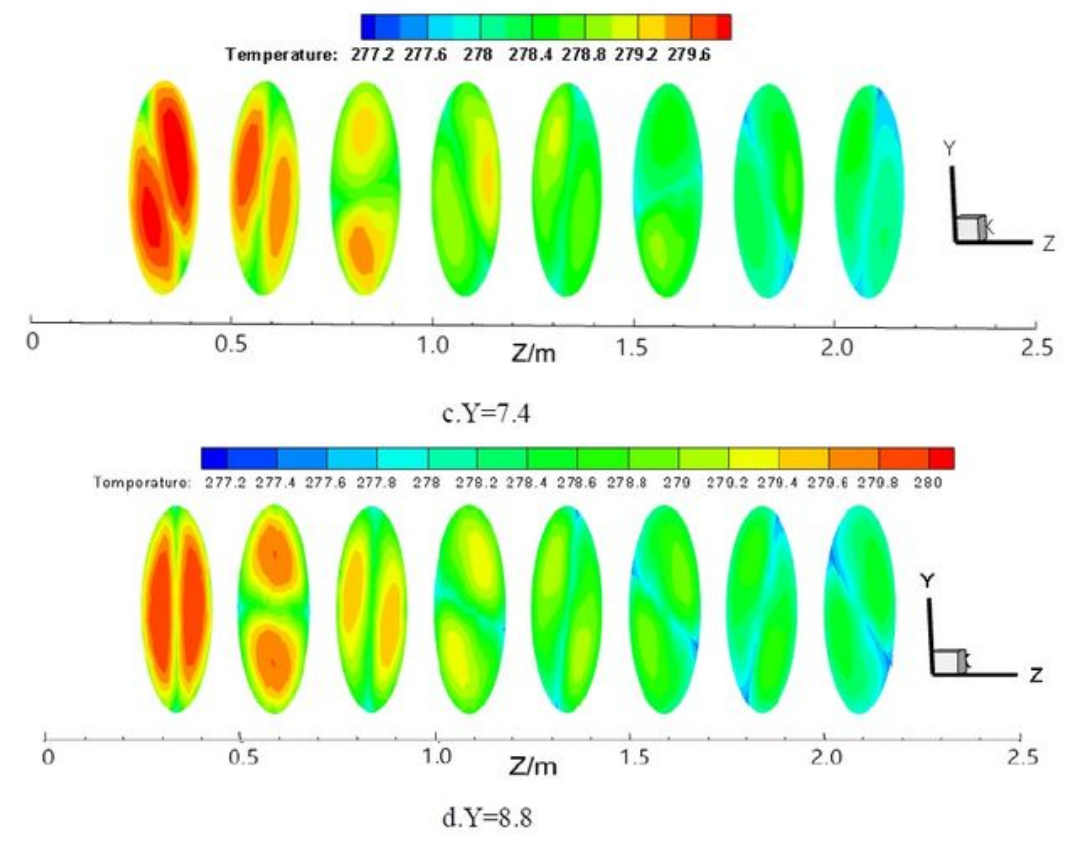

Figure 12

Temperature nephogram of each section in long twisted band pipeline with different twist ratio at $\mathrm{Re}=20000$ 


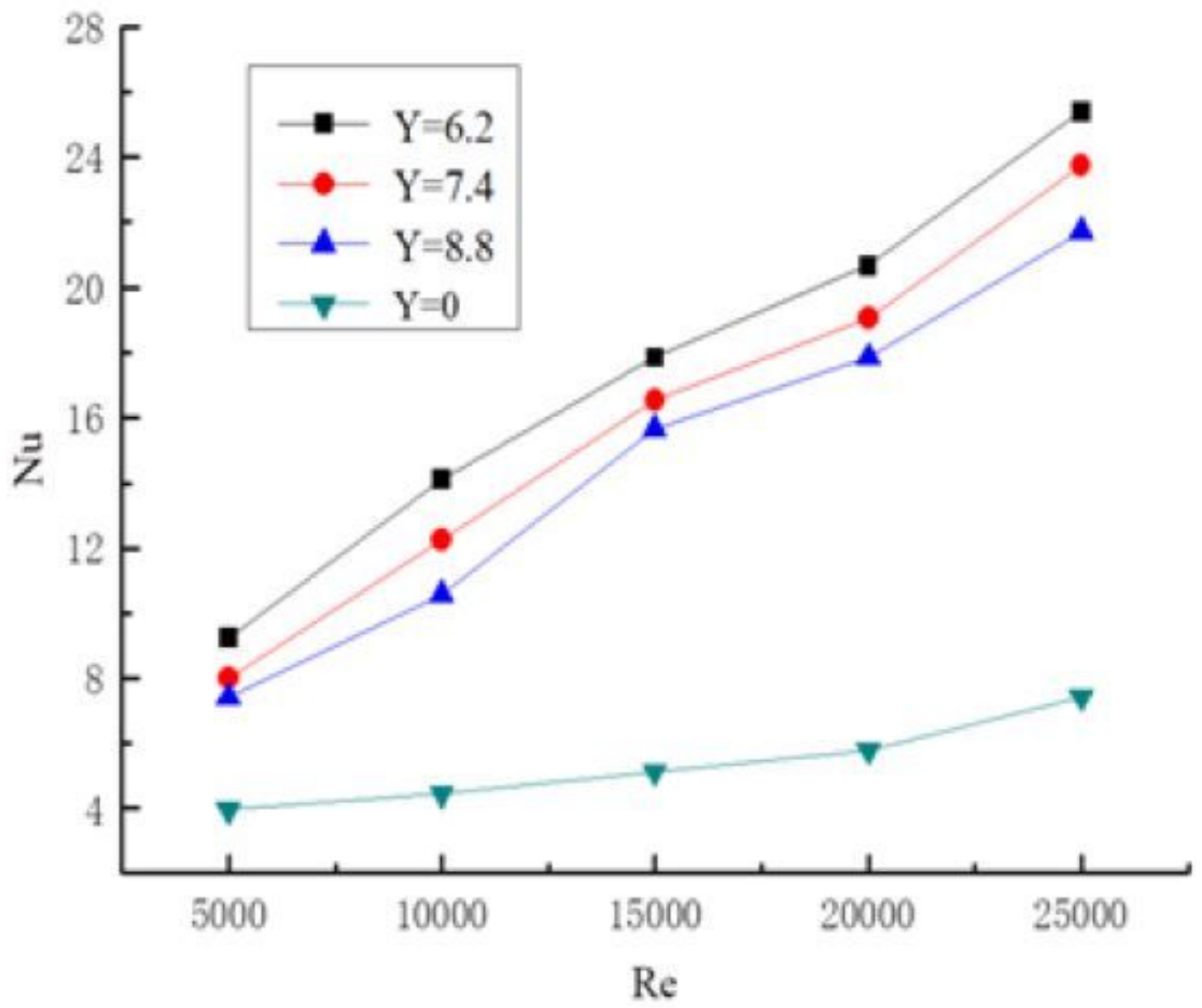

Figure 13

Variation curve of nussel number with $\mathrm{Re}$ in long twisted band pipeline 


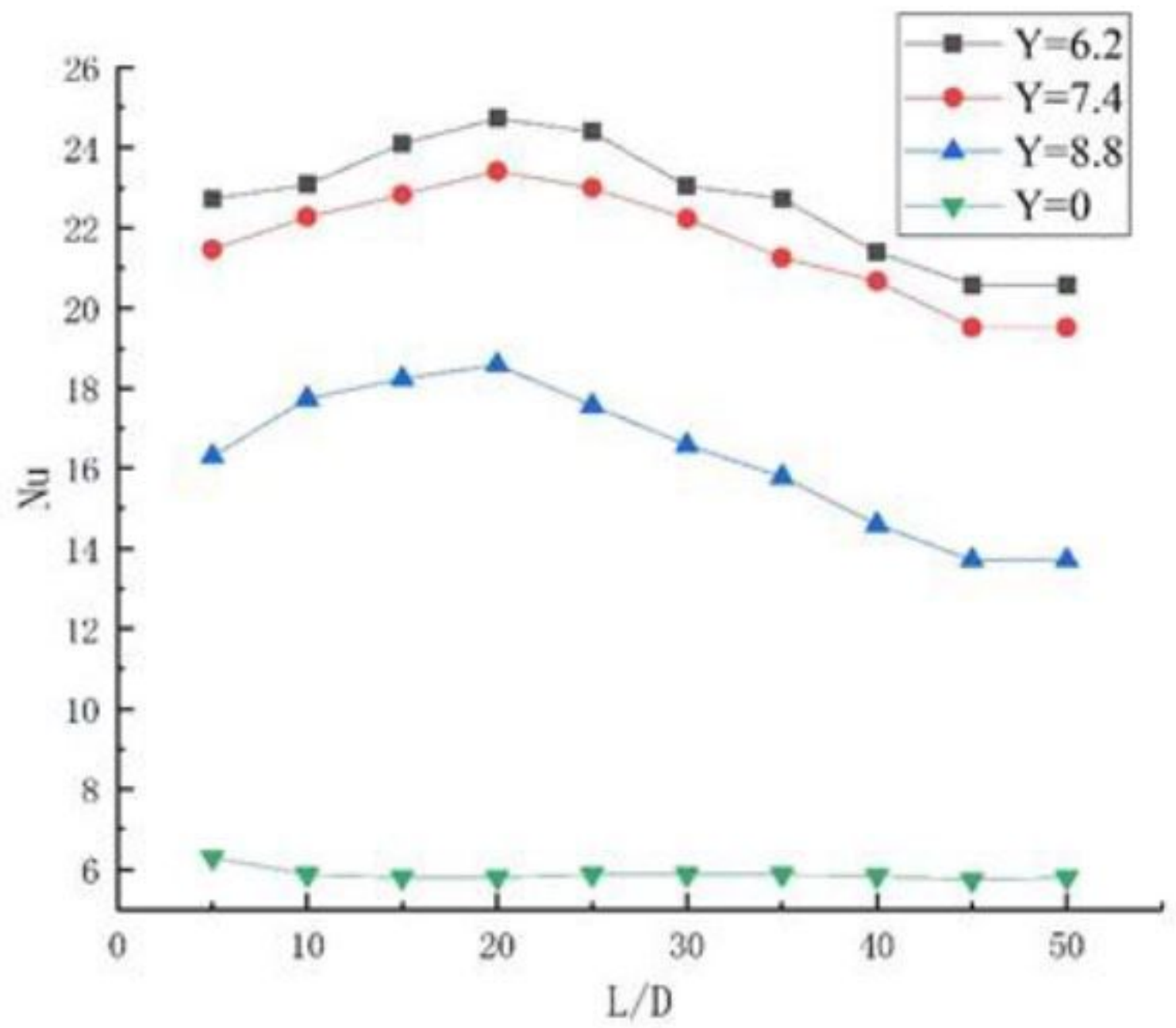

Figure 14

Nussel number distribution curves of different sections under different twist rates at $\mathrm{Re}=20000$ 


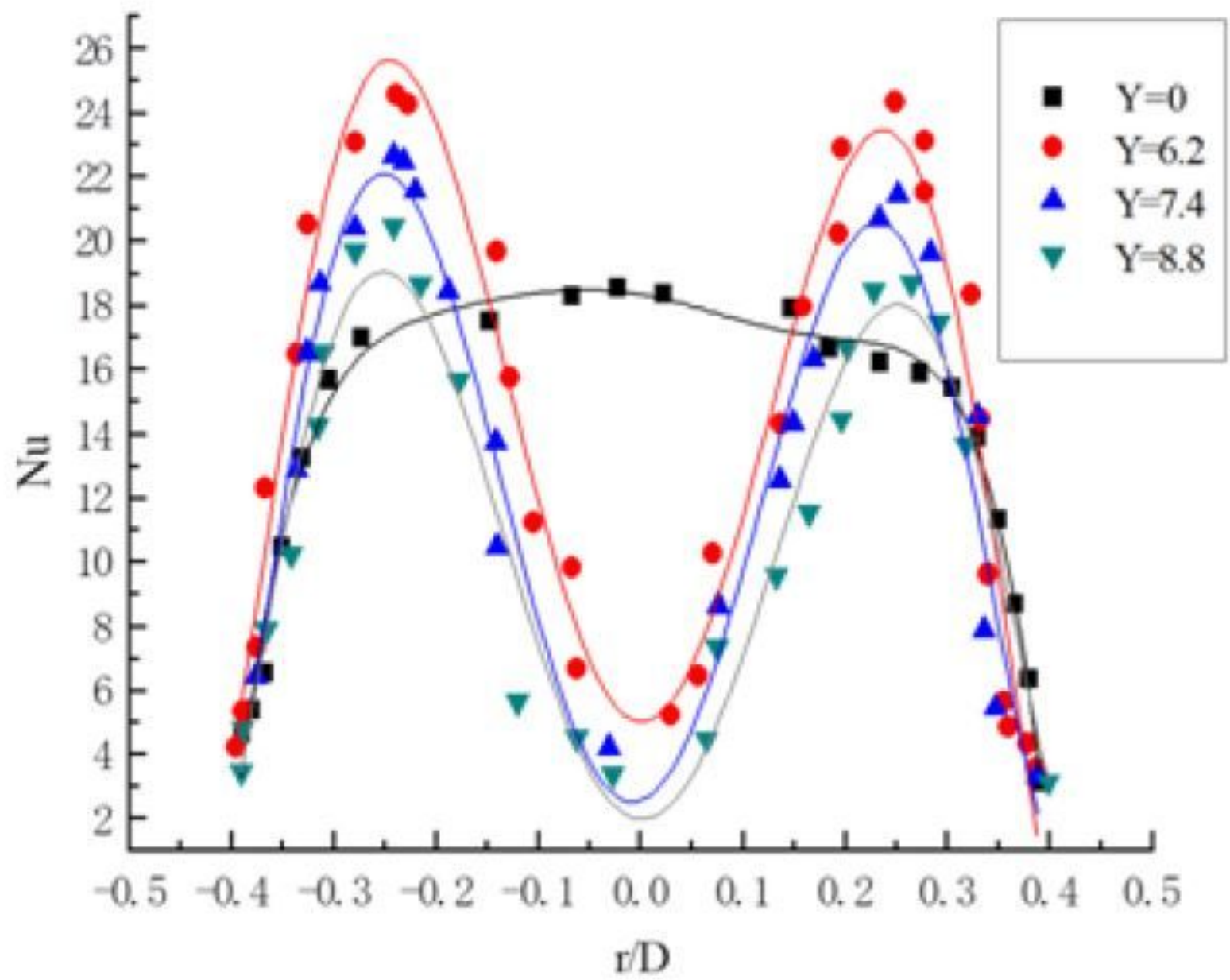

Figure 15

Nussel number distribution curve on $Z=5 D$ section at $R e=20000$ 
DPM-Conc: $0.00 \quad 0.290 .57 \quad 0.861 .141 .431 .712 .002 .292 .572 .863 .143 .43 \quad 3.714 .00$

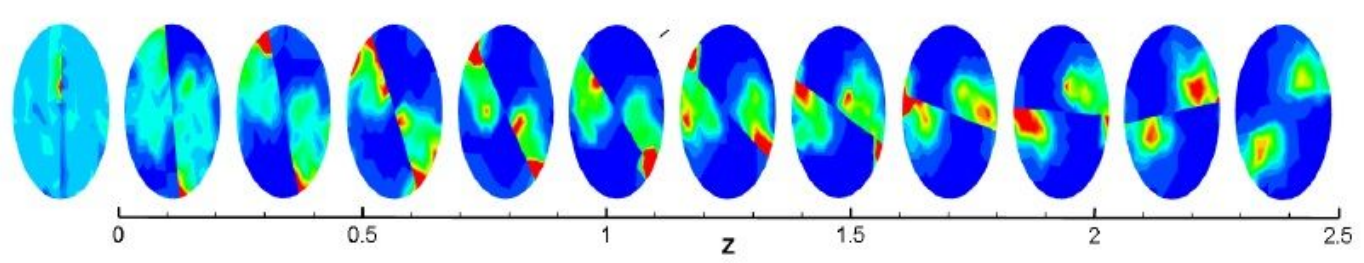

(a) Long twist band $\mathrm{Y}=6.2$

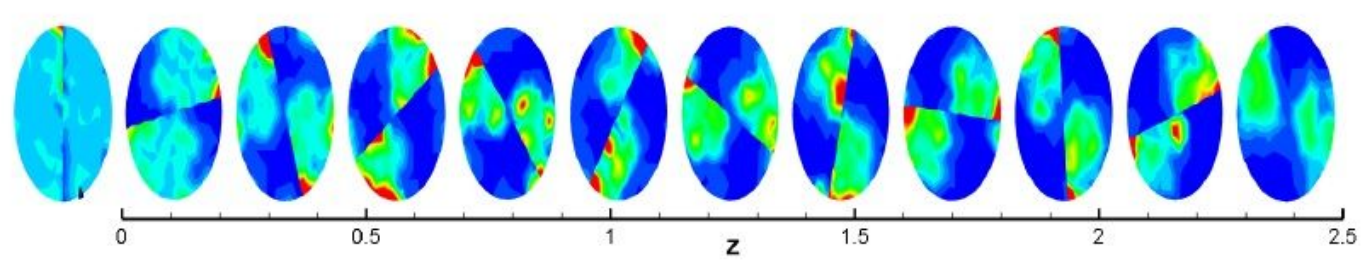

(b) Long twist band $\mathrm{Y}=7.4$

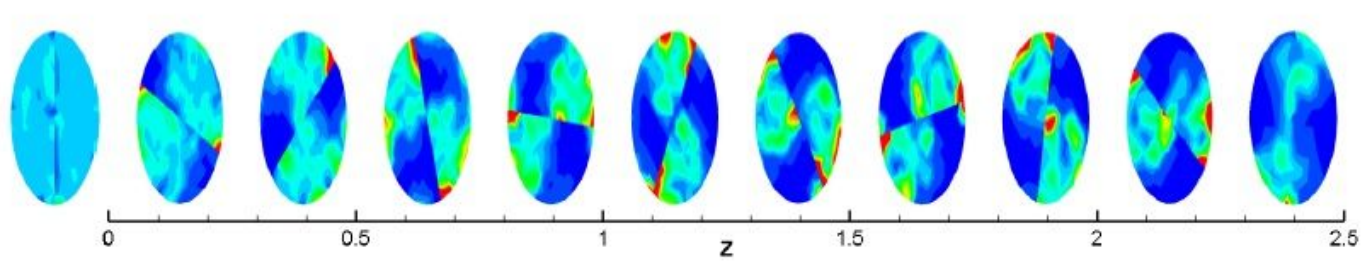

(c) Long twist band $\mathrm{Y}=8.8$

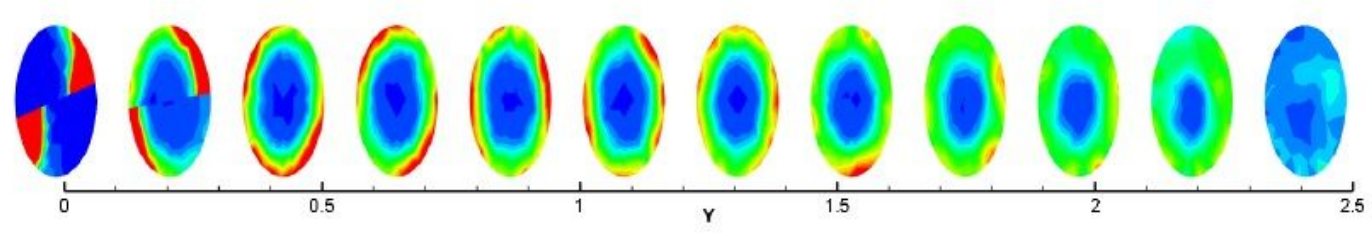

(d) Short twist band $\mathrm{Y}=6.2$

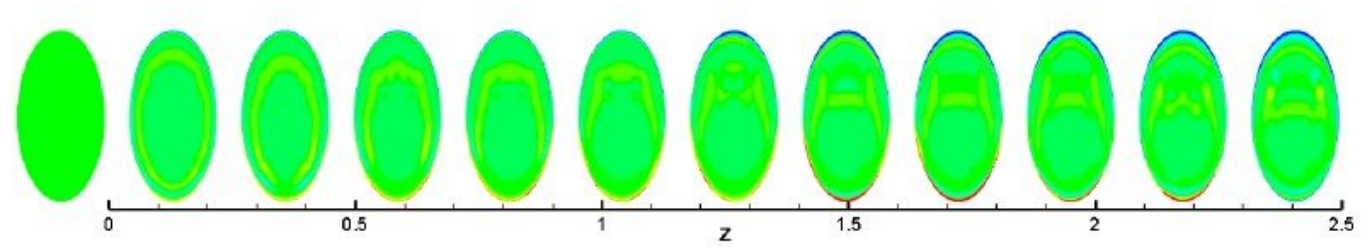

(e) No twist band

\section{Figure 16}

Distribution of particle concentration at different sections of the pipeline 


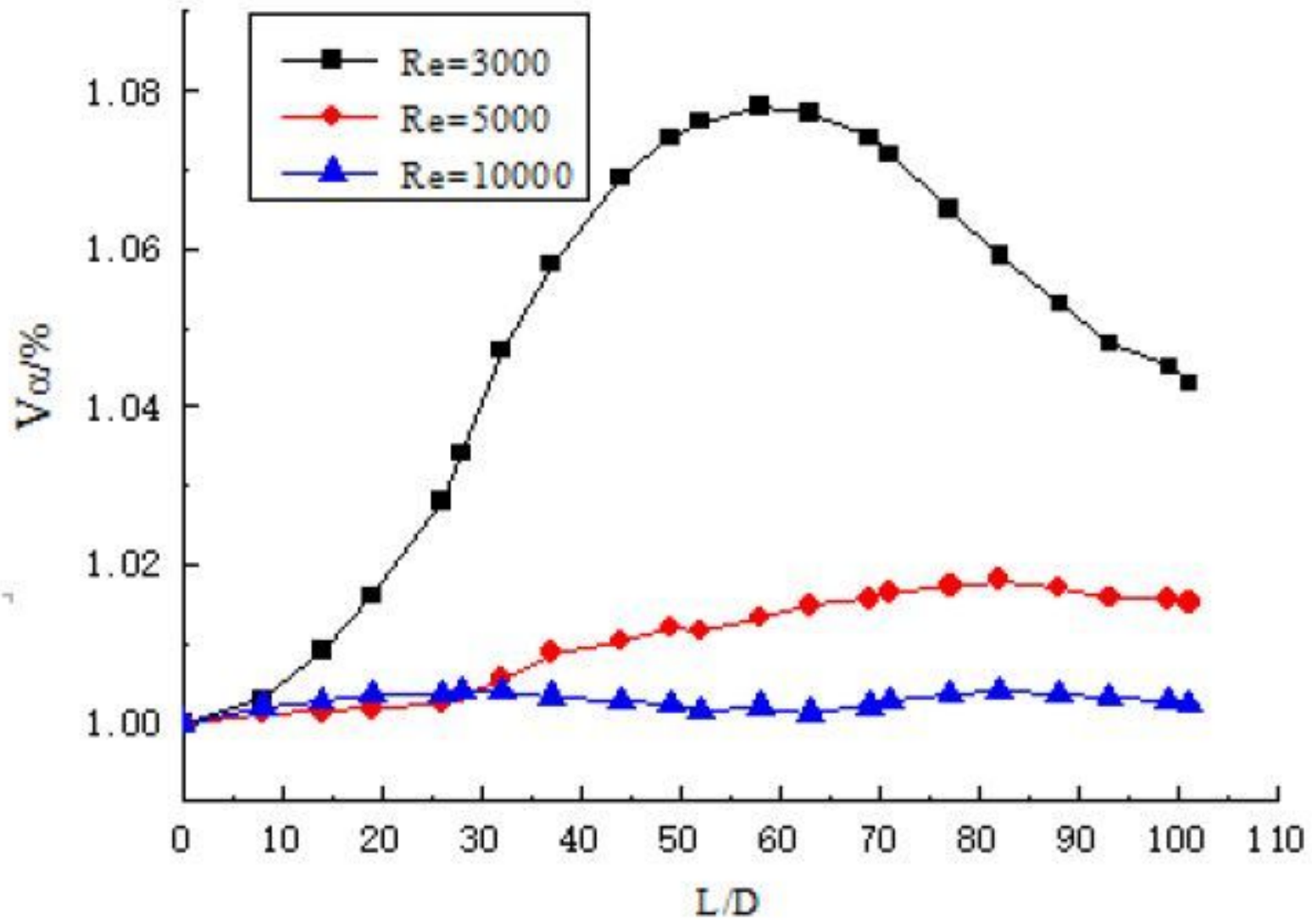

Figure 17

Volume fraction distribution curve of particles along pipeline at $\mathrm{Y}=6.2$ 


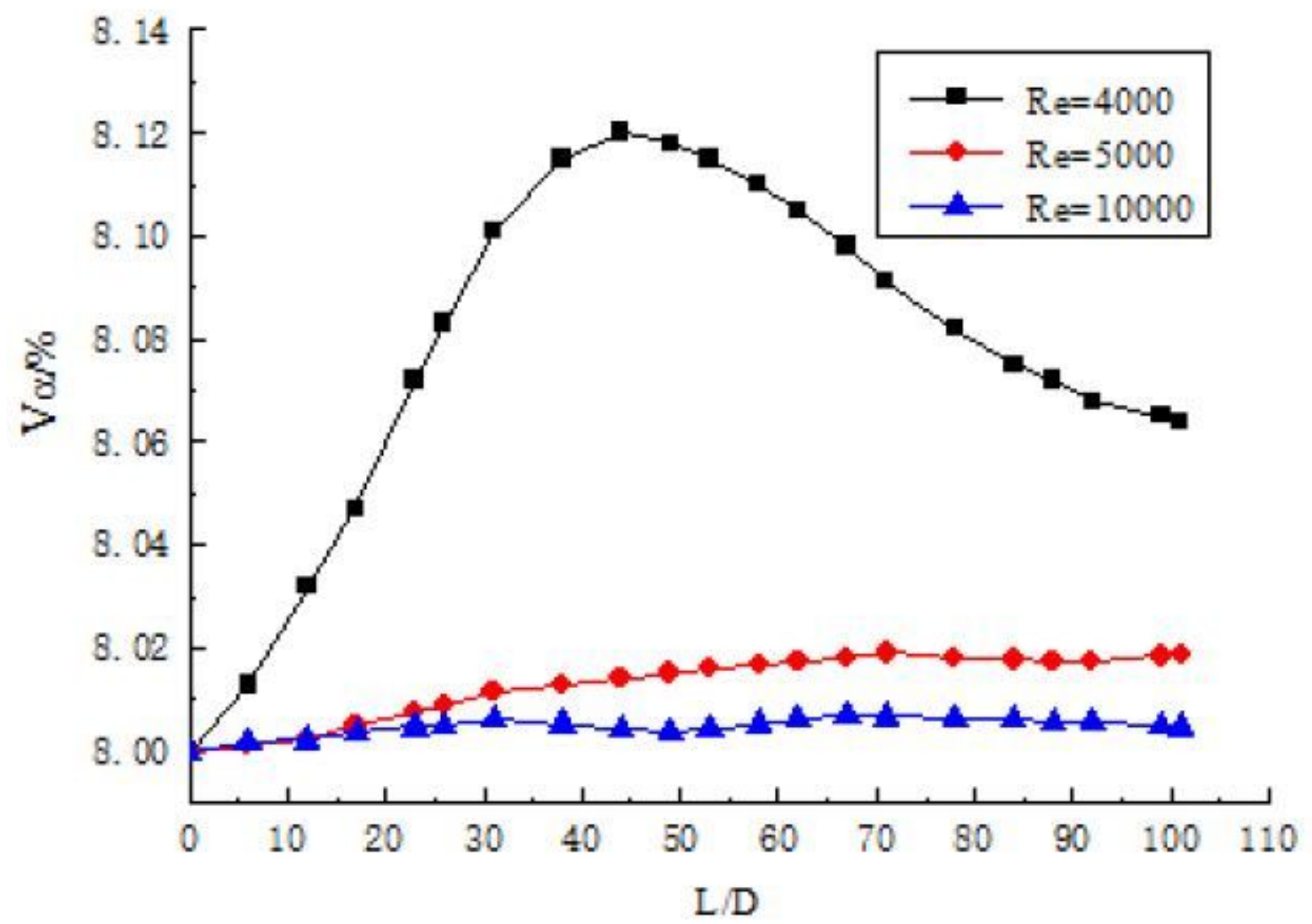

Figure 18

Volume fraction distribution curve of particles along pipeline at $Y=6.2, a 0=8 \%$ 


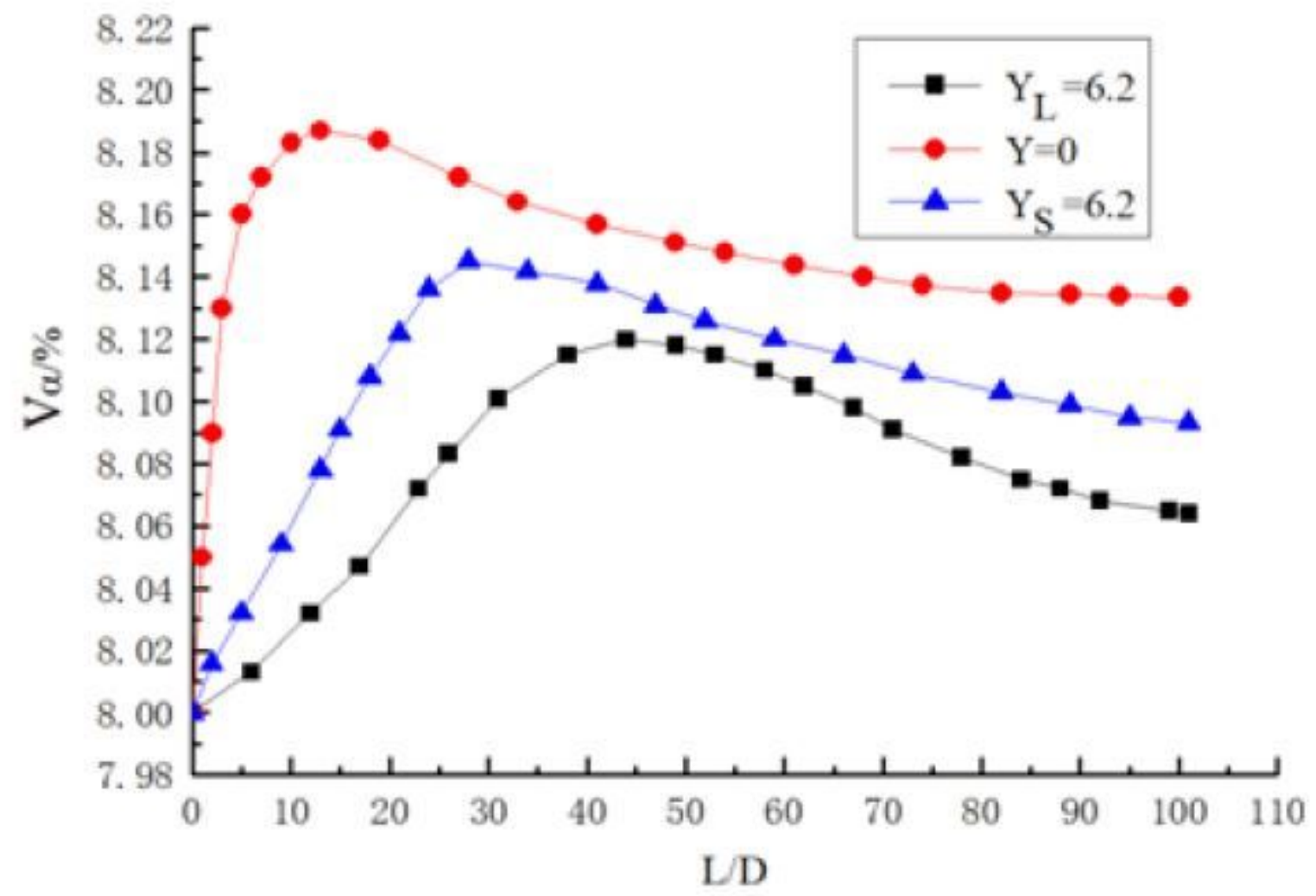

Figure 19

Volume fraction distribution curve of different twisted band particles along pipeline at $\operatorname{Re}=4000, a 0=8 \%$ 